\title{
Classification of unitary vertex subalgebras and conformal subnets for rank-one lattice chiral CFT models ${ }^{\circledR}$
}

Cite as: J. Math. Phys. 60, 093505 (2019); https://doi.org/10.1063/1.5094581

Submitted: 04 March 2019 . Accepted: 01 September 2019. Published Online: 20 September 2019

Sebastiano Carpi (D), Tiziano Gaudio, and Robin Hillier (D)

COLLECTIONS

EP This paper was selected as an Editor's Pick

\section{AVS Quantum Science}

A high impact interdisciplinary 


\title{
Classification of unitary vertex subalgebras and conformal subnets for rank-one lattice chiral CFT models
}

\author{
Cite as: J. Math. Phys. 60, 093505 (2019); doi: 10.1063/1.5094581 \\ Submitted: 4 March 2019 - Accepted: 1 September 2019• \\ Published Online: 20 September 2019
}

Sebastiano Carpi, ${ }^{1, a)}$ (D) Tiziano Gaudio, ${ }^{2, b)}$ and Robin Hillier ${ }^{2, c)}$ (D)

\begin{abstract}
AFFILIATIONS
${ }^{1}$ Dipartimento di Economia, Università di Chieti-Pescara "G. d'Annunzio", Viale Pindaro, 42, 65127 Pescara, Italy

${ }^{2}$ Department of Mathematics and Statistics, Lancaster University, Lancaster LA1 4YF, United Kingdom

a) E-mail: s.carpi@unich.it

b) E-mail: t.gaudio@lancaster.ac.uk

${ }^{c}$ E-mail: r.hillier@lancaster.ac.uk
\end{abstract}

\begin{abstract}
We provide a complete classification of unitary subalgebras of even rank-one lattice vertex operator algebras. As a consequence of the correspondence between vertex operator algebras and conformal nets, we also obtain a complete classification of conformal subnets of even rank-one lattice conformal nets.
\end{abstract}

Published under license by AIP Publishing. https://doi.org/10.1063/1.5094581

\section{INTRODUCTION}

Two-dimensional conformal quantum field theory (2D CFT) is an exciting topic, both from the physical and from the mathematical point of view, with a variety of applications, cf. Refs. 14, 31, and 34 for some of them. The special subclass of chiral CFTs, namely, those 2D CFTs containing right-moving fields only or left-moving fields only, are the building blocks of 2D CFT. These theories can be considered as quantum field theories on the real line (the light-ray) or, after compactification, on the circle $S^{1}$.

There are mainly two mathematical axiomatizations of chiral CFT, one of them goes via conformal nets and the other one via vertex operator algebras (VOAs).

A conformal net is a family of von Neumann algebras indexed by the intervals of $S^{1}$. These algebras should be regarded as the algebras generated by bounded versions of smeared fields localized in the respective interval. Moreover, the family should satisfy a number of axioms motivated by physics; in particular, it should carry an action of the (orientation-preserving) diffeomorphism group Diff ${ }^{+}\left(S^{1}\right)$. The overall framework in this approach is that of algebraic quantum field theory (AQFT), cf. Ref. 39 for an overview.

The second approach goes via vertex operator algebras. A vertex operator is a certain formal Laurent series and is thought of as a quantum field. Together they give rise to a vertex operator algebra, which formally consists of a vector space together with a state-field correspondence and some additional structure necessary to make things work. This additional structure is an axiomatization of physical properties, but the axioms here are different from the ones of conformal nets and can be seen rather as an algebraic formulation of the Wightman axioms; see, e.g., Ref. 43.

Various problems that can be solved in the conformal net approach are still open in the VOA approach and vice versa. It is generally believed that these two frameworks should be more or less equivalent in the unitary case, but for a long time a direct general connection between those two approaches had not been clear. However, a significant step forward has been achieved recently in Ref. 10, where a map from a suitable class of unitary VOAs, the class of strongly local VOAs, to the class of conformal nets has been defined. Moreover, many examples of unitary VOAs have been shown to be strongly local and it is conjectured that the map gives in fact a one-to-one correspondence between (simple) unitary VOAs and (irreducible) conformal nets; see also Ref. 45 for an overview of these results and related 
conjectures. Further progress in the connection of these two mathematical frameworks for chiral CFT has been made in Refs. 11, 37, 38, 54, and 55.

In both approaches, there is a natural notion of subtheory of a given chiral CFT. These subtheories are called conformal subnets or unitary vertex subalgebras (or simply unitary subalgebras) depending on the chosen framework. For a conformal net $\mathcal{A}_{V}$ that comes from a strongly local VOA $V$, it has been shown in Ref. 10 that the conformal subnets $\mathcal{A} \subset \mathcal{A}_{V}$ are in one-to-one correspondence with the unitary subalgebras $W \subset V$. The study and the classification of these subtheories is a very natural problem in either of the two approaches. One of the reasons is that subtheories give rise to new models from known ones. Typical examples are the orbifold models or the coset models, but the study of subtheories could give new exotic examples besides these standard constructions. For example, Evans and Gannon in Ref. 30 argued in favor of the existence of "exotic" subtheories of certain affine Lie algebra chiral CFT models that should be related in an appropriate sense to the Haagerup subfactor.

In the AQFT setting, the structure of (Haag dual) subnets of a given local Poincaré covariant net on the four-dimensional Minkowski space-time is quite well understood under rather mild and natural assumptions. ${ }^{6-8,12}$ Here, the subnet structure can be completely described in terms of fixed point subnets under actions of compact groups of inner symmetries (compact orbifolds) and tensor product decompositions, after replacing the net, if necessary, with its maximal extension, namely, the canonical field net of Doplicher and Roberts. ${ }^{29}$ Moreover, starting from the general description, one can describe the subnet structure in a very explicit way for many free field models. These results rely heavily on Doplicher-Roberts abstract duality for compact groups, ${ }^{27,28}$ on which the construction of the canonical field net in Ref. 29 is based. In particular, they rely on the permutation symmetry of the superselection sectors, and hence, they do not apply in low space-time dimensions because of the presence of nontrivial braid group relations.

In the conformal net setting, there are various abstract results on the general structure of conformal subnets, see, e.g., Refs. 1, 9, 13, 41, 49,50 , and 57, but it is not clear how to use them in order to get explicit classification results in concrete models. For example, it has been shown in Ref. 1 that the finite index subnets of a given net can be completely described in terms of hypergroup actions, but it is not clear how to determine the structure of these hypergroup actions without knowing the subnet structure $a$ priori.

Actually, only few results appear to be known for the classification of conformal subnets in concrete models, cf. Refs. 3 and 4 and (Ref. 46, Subsection 6.3). In more detail, it is known that a Virasoro net has no proper nontrivial conformal subnet ${ }^{3}$ that all the nontrivial subnets of the loop group net associated with the level one vacuum representation of the loop group for SU(2) arise as compact orbifolds for closed subgroups of the automorphism group $\mathrm{SO}(3)$ of the net. ${ }^{4}$ Moreover, the finite index conformal subnets of all the conformal nets with central charge $c<1$ are classified in Ref. 46 . The results in Refs. 3 and 4 have a (unitary) VOA analog; ${ }^{10}$ see also Ref. 17. This is not surprising in view of the fact than the underlying VOAs are known to be strongly local so one can directly apply the correspondence between conformal subnets and unitary subalgebras. Concerning the unitary VOAs with $c<1$, strong locality results are presently available only in some special cases, but it should be possible to obtain the analogous results of Ref. 46, Subsection 6.3 directly from the classification of preunitary VOAs with $c<1$ given in Ref. 23.

One immediate example of a $c=1$ model consists of the $\mathrm{U}(1)$-current algebra CFT, also called free boson CFT. Its extensions have been classified in Ref. 2 in the conformal net setting. They can be expressed as the conformal nets $\mathcal{A}_{\mathrm{U}(1)_{2 N}}$ or as the simple unitary VOAs $V_{L_{2 N}}$, respectively, associated with an even rank-one lattice $\alpha \mathbb{Z}$ with generator $\alpha$ satisfying $(\alpha \mid \alpha)=2 N$, where $N \in \mathbb{Z}_{>0}$ labels the lattices uniquely. These models have been studied, e.g., in Refs. 2, 56, and 26 or Refs. 16 and 17, respectively.

It is widely believed that these models together with their (compact, possibly finite) orbifolds exhaust all possible unitary chiral CFTs with $c=1$; see, e.g., Refs. 15, 35, and 47. This fact has been proved in the conformal net setting by Xu ${ }^{56}$ under an assumption called "Spectrum Condition;" see also Ref. 5 for some special cases of these results. This assumption is verified in all the above $c=1$ conformal nets, but its general validity is still an open problem. On the VOA side, recent progress on the classification of rational unitary $c=1$ chiral CFTs has been made in Refs. 19 and 20, but again the problem is still open.

In this paper, we completely classify the conformal subnets and the unitary vertex subalgebras of all currently known chiral CFT models with central charge $c=1$. We first give the classification in the unitary VOA framework. We only use (unitary) VOA arguments without reference to strong locality or conformal nets. To this end, we rely on previous results by Dong and Griess. ${ }^{17}$ We show that if $N$ is not the square of a positive integer and $N \neq 2$, then every nontrivial unitary vertex subalgebra of $V_{L_{2 N}}$ is either an orbifold $V_{L_{2 N}}^{H}$ for some closed subgroup of the compact group of unitary VOA automorphisms $D_{\infty}$ of $V_{L_{2 N}}$ or it coincides with the Virasoro subalgebra generated by the $c=1$ conformal vector. If $N=2$, besides the compact orbifolds and the $c=1$ Virasoro subalgebra, there is a further explicitly described continuous family $\left\{W_{t}: t \in \mathbb{T}\right\}$ of unitary subalgebras, all isomorphic to the $c=1 / 2$ simple Virasoro VOA, but there are no further nontrivial

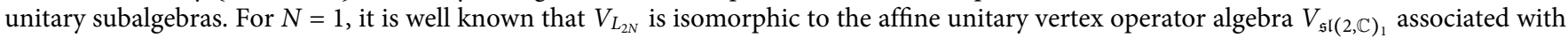
$\mathfrak{s l}(2, \mathbb{C})$ at level one. We show that every nontrivial unitary subalgebra is an orbifold $V_{L_{2}}^{H}$ for some closed subgroup of the compact group of unitary VOA automorphisms $\mathrm{SO}(3)$ of $V_{L_{2}}$. As already mentioned, the latter result was already known, but our proof is slightly different from the one given in Ref. 10 in order to have a purely VOA argument. Finally, for every integer $k>1$, the classification of the unitary subalgebras $V_{L_{2 k^{2}}}$ follows from the unitary embedding $V_{L_{2 k^{2}}} \subset V_{L_{2}}$. The results on the classification of conformal subnets are then obtained using the correspondence between simple unitary subalgebras and conformal subnets given in Ref. 10 and the fact that the rank-one lattice VOAs are known to be strongly local. In particular, our results show that no new $c=1$ chiral CFT model can be found by taking subtheories of the currently known $c=1$ chiral CFTs, in agreement with the common belief.

This paper is organized as follows: We start in Sec. II by recalling some basics about unitary VOAs in general and the rank-one lattice type models, in particular. Section III deals with the main part of the classification in the VOA setting. Finally, Sec. IV translates those results into the language of conformal nets, recalling and using the correspondence between the two settings developed in Ref. 10. 


\section{PRELIMINARIES}

This section is dedicated to setting the notation we use throughout the paper and to introducing some preliminaries about unitary vertex operator algebras (VOAs) and even rank-one lattice type VOAs. References are given at the beginning of every subsection when required.

\section{A. Unitary vertex operator algebras}

The present introduction to unitary vertex operator algebras follows mostly the structure and the notation given in Ref. 10 , Secs. 4 and 5; see also Ref. 48, Chaps. 2 and 6, and Ref. 43, Chap. 4.

Given a vector space $V$ on $\mathbb{C}$, consider the space of doubly infinite formal Laurent series in the variable $z$ on $\mathbb{C}$ with coefficients in End $V$ (see Ref. 48, Chap. 2),

$$
(\text { End } V)\left[\left[z, z^{-1}\right]\right]:=\left\{a(z):=\sum_{n \in \mathbb{Z}} a_{(n)} z^{-n-1} \mid a_{(n)} \in \operatorname{End} V\right\} \text {. }
$$

Then, a vertex algebra is defined by a quadruple $(V, \Omega, Y, T)$ :

- $\Omega \in V$ is called the vacuum vector of $V$.

- $T \in$ End $V$ is called the infinitesimal translation operator of $V$.

- $Y$ is a linear map between $V$ and $($ End $V)\left[\left[z, z^{-1}\right]\right]$, called the state-field correspondence, defined by

$$
a \longmapsto Y(a, z):=\sum_{n \in \mathbb{Z}} a_{(n)} z^{-n-1}
$$

with the following properties:

1 (Field). For any $a \in V, Y(a, z)$ is a field; namely, for all $b \in V$, there exists an integer $N \geq 0$ such that $a_{(n)} b=0$ for all $n \geq N$.

2 (Translation covariance). $[T, Y(a, z)]=\frac{\mathrm{d}}{d z} Y(a, z)$ for all $a \in V$.

3 (Vacuum). $T \Omega=0, Y(\Omega, z)=I_{V}, a_{(-1)} \Omega=a$ for all $a \in V$.

4 (Locality). For all $a, b \in V$, there exist $N>0$ such that (see [43, Chapter 2] for formal calculus for fields)

$$
(z-w)^{N}[Y(a, z), Y(b, w)]=0 .
$$

We call such a field $Y(a, z)$ a vertex operator.

A vertex operator algebra or just $V O A$ is a vertex algebra $(V, \Omega, Y, T)$ with an element $v \in V$, called the conformal vector of $(V, \Omega, Y, T)$, such that

- $v$ is a Virasoro vector with central charge $c \in \mathbb{C}$, which means that $Y(v, z)=\sum_{n \in \mathbb{Z}} L_{n} z^{-n-2}$, where $L_{n}:=v_{(n+1)}$ is a Virasoro field, i.e., the endomorphisms $L_{n}$ satisfy the following commutation relations:

$$
\left[L_{m}, L_{n}\right]=(m-n) L_{m+n}+\frac{c\left(m^{3}-m\right)}{12} \delta_{m,-n} \quad \forall m, n \in \mathbb{Z}
$$

- $v$ is a conformal vector, which means that $L_{-1}=T$ and $L_{0}$ is diagonalizable on $V$. In this case, $Y(v, z)$ and $L_{0}$ are called the energymomentum field and the conformal Hamiltonian of $(V, \Omega, Y, T, v)$, respectively.

- The following conditions must hold:

$1 V=\bigoplus_{n \in \mathbb{Z}} V_{n}$, where $V_{n}:=\operatorname{Ker}\left\{L_{0}-n I_{V}\right\} ;$

$2 \operatorname{dim} V_{n}<\infty$;

3 there exists an $N \in \mathbb{Z}$ such that $V_{n}=0$ for all $n \leq N$.

We will often indicate a $\operatorname{VOA}(V, \Omega, Y, T, v)$ just with the vector space $V$.

An antilinear/linear automorphism $g$ of a VOA $V$ is a vector space antilinear/linear automorphism such that

$$
g(\Omega)=\Omega, \quad g(v)=v, \quad g\left(a_{(n)} b\right)=g(a)_{(n)} g(b) \quad \forall a, b \in V .
$$

We define Aut $V$ the group of linear automorphisms of $V$, which has a natural structure of a topological group (see Ref. 10, Sec. 4.3). In a similar manner, one defines a linear isomorphism between two VOAs.

A VOA $V$ is of CFT type if $V_{n}=0$ for all $n<0$ and $V_{0}=\mathbb{C} \Omega$.

If $a \in V_{n}$, we say that $a$ is homogeneous of conformal weight $d_{a}:=n$. In this case, we also use the following notation: 


$$
Y(a, z)=\sum_{n \in \mathbb{Z}} a_{n} z^{-n-d_{a}}, \quad a_{n}:=a_{\left(n+d_{a}-1\right)}
$$

We call an element $a \in V$ primary if $L_{n} a=0$ for all $n \geq 1$ and quasiprimary if $L_{1} a=0$. Therefore, using the fact that (see Ref. 10, Sec. 4.1)

$$
a_{(n)} \Omega=0, \quad a \in V, n \geq 0
$$

we have that $\Omega$ is a primary vector in $V_{0}$. Furthermore, it is possible to prove that $v \in V_{2}$ and that it is a quasiprimary vector which is not primary if $c \neq 0$ (see Ref. 10 , Secs. 4.1 and 4.2 ).

To define a unitary structure on a VOA $V$, we recall some facts and definitions, which the reader may find in more detail in Ref. 10, Secs. 4.3 and 5.1 and Ref. 32, Sec. 5.2. First of all, we say that a bilinear form $(\cdot, \cdot)$ on $V$ is invariant if

$$
(Y(a, z) b, c)=\left(b, Y\left(e^{z L_{1}}\left(-z^{-2}\right)^{L_{0}} a, z^{-1}\right) c\right), \quad a, b, c \in V
$$

In particular, $\left(a, L_{n} b\right)=\left(L_{-n} a, b\right)$ for all $n \in \mathbb{Z}$. Consequently, we say that a scalar product $(\cdot \mid \cdot)$ on $V$ (a positive-definite sesquilinear form, linear in the second variable) is invariant if there exists an antilinear automorphism $\theta$ of $V$, called a PCT operator for $V$, which makes $(\theta(\cdot) \mid \cdot)$ an invariant bilinear form. Moreover, a scalar product on $V$ is said to be normalized if $(\Omega \mid \Omega)=1$. We point out (see Ref. 10, Proposition 5.1) that for every normalized invariant scalar product on a VOA $V$, there exists a unique PCT operator.

We can now define a unitary vertex operator algebra as a VOA $V$ equipped with an invariant normalized scalar product $(\cdot \mid \cdot)$. In this case, we say that a linear automorphism $g$ is unitary if $(g(a) \mid g(b))=(a \mid b)$ for all $a, b \in V$, and we define Aut $(\cdot \mid \cdot) V$ as the group of unitary linear automorphisms; it is a compact subgroup of Aut $V$ (see Ref. 10, Lemma 5.20). Similarly, one defines a unitary isomorphism between two unitary VOAs.

An ideal of a vertex algebra $V$ is a $T$-invariant vector subspace $\mathcal{I} \subseteq V$ such that $a_{(n)} b \in \mathcal{I}$ for all $a \in V, b \in \mathcal{I}$, and $n \in \mathbb{Z}$. By [Ref. 43, (4.3.1)], we also have $b_{(n)} a \in \mathcal{I}$. A vertex algebra $V$ is simple if $V$ and $\{0\}$ are the only ideals. Furthermore, by Ref. 10, Proposition 5.3, a unitary VOA $V$ is simple if and only if $V_{0}=\mathbb{C} \Omega$. Consequently, every simple unitary VOA is of CFT type (cf. Ref. 10, Remark 4.5).

A vertex subalgebra of a vertex algebra $V$ is a vector subspace $W$ of $V$ such that $\Omega \in W$ and $a_{(n)} b \in W$ for all $a, b \in W$. By Ref. 10, p. 24, every vertex subalgebra $W$ is $T$-invariant; thus, $W$ is a vertex algebra too. Then, we have the following definition:

Definition 2.1. A unitary vertex subalgebra of a unitary VOA $V$ with PCT operator $\theta$ is a vertex subalgebra $W$ of $V$ such that $\theta(W) \subseteq W$ and $L_{1}(W) \subseteq W$. For convenience, we call a unitary vertex subalgebra simply a unitary subalgebra.

For convenience, we have chosen to define unitary subalgebras by Definition 2.1 instead of the standard one (Ref. 10, Definition 5.22). In any case, these are equivalent, thanks to Ref. 10, Proposition 5.23. Note also that, by Ref. 10, Definition $5.22, L_{0}(W) \subseteq W$ for any unitary subalgebra $W$.

Let $S$ be a subset of a unitary VOA $V$. We indicate with $W(S)$ the smallest vertex subalgebra of $V$ containing $S$, and we say that $S g e n e r a t e s$ a vertex subalgebra of $V$. Moreover, if $W(S)$ is a unitary subalgebra as in Definition 2.1, then we say that $S$ generates a unitary subalgebra of $V$.

For further use, we state the following results about unitary subalgebras from Ref. 10, Proposition 5.29.

Proposition 2.2. Let $(V, \Omega, T, Y, v,(\cdot \mid \cdot))$ be a simple unitary VOA and $W$ be a unitary subalgebra of $V$. Consider the projection $e_{W}$ into $W$ and set $\omega:=e_{W}(v)$ with $Y(\omega, z)=\sum_{n \in \mathbb{Z}} L_{n}^{\omega} z^{-n-2}$ the corresponding field. Then,

(i) $\theta(\omega)=\omega$.

(ii) $\quad \omega$ is a Virasoro vector of $V$ and $\left.L_{n}^{\omega}\right|_{W}=\left.L_{n}\right|_{W}$ for $n \in\{-1,0,1\}$.

(iii) $\quad \omega$ is a conformal vector for $W$, and $(W, \Omega, T, Y, \omega,(\cdot \mid \cdot))$ is a simple unitary VOA with PCT operator $\left.\theta\right|_{W}$.

Now, some classical examples of unitary VOAs.

Example 2.3. For every unitary VOA $V$, the vector subspace $\mathbb{C} \Omega$ is a unitary subalgebra, which we call the trivial subalgebra. Recall that $T \Omega=0$ and $Y(\Omega, z)=I_{V}$. Furthermore, $e_{\mathbb{C} \Omega}(v)=0,(a \Omega \mid b \Omega)=\bar{a} b$, and $\theta(a \Omega)=\bar{a} \Omega$ for all $a, b \in \mathbb{C}$.

Example 2.4. (See Ref. 10, Example 5.25). Let $G \subseteq \operatorname{Aut}_{(\cdot \mid \cdot)} V$ be a closed subgroup. Then,

$$
V^{G}:=\{v \in V \mid g(v)=v \quad \forall g \in G\}
$$


is a unitary subalgebra of $V$, called fixed point subalgebra. Moreover, if $G$ is finite, $V^{G}$ is called the orbifold subalgebra.

Example 2.5. By Ref. 10, Example 5.24, the conformal vector $v$ with central charge $c$ of a unitary VOA always generates a simple unitary subalgebra, called the Virasoro subalgebra of $V$, indicated with $L(c, 0) \subset V$. The admissible values of $c \in \mathbb{C}$ are the following ones:

$$
\begin{gathered}
c \geq 1, \\
c_{m}=1-\frac{6}{m(m+1)}, m \geq 2 .
\end{gathered}
$$

Moreover, $L(c, 0)$ is a unitary VOA called the unitary Virasoro VOA with central charge $c$, which can be directly constructed from the Virasoro Lie algebra of central charge $c$, namely, the Lie algebra with formal generators $L_{n}$ and 1 and commutation relations (1), cf. Ref. 43, Example 4.10; Ref. 48, Sec. 6.1; and Ref. 44, Lecture 3, 12.3 for the construction and Ref. 22, Sec. 4.1, and Ref. 10, Example 5.6 for the unitary structure. The irreducible modules of $L(c, 0)$ are denoted by $L(c, h)$ and arise from unitary irreducible positive-energy representations of the Virasoro Lie algebra with central charge $c$; $h$ denotes the lowest eigenvalue of $L_{0}$ on that module. The admissible values of $c$ and $h$, which completely determine each unitary irreducible positive-energy representation, are

$$
\begin{array}{rll}
c \geq 1, & \text { and } & h \geq 0, \\
c_{m}=1-\frac{6}{m(m+1)}, & \text { and } & h_{p, q}(m)=\frac{((m+1) p-m q)^{2}-1}{4 m(m+1)}
\end{array}
$$

for all $m \geq 2$ and $1 \leq q \leq p \leq m-1$, cf. also Ref. 44 , Lecture 8.4 .

Example 2.6. By Ref. 10, Example 5.26, we have that a family of $\theta$-invariant quasiprimary vectors in a unitary VOA $V$ generates a unitary subalgebra. Thus, if $W$ is a unitary subalgebra of $V$, the conformal vector $\omega=e_{W}(v)$ as in Proposition 2.2 generates a unitary subalgebra of $V$ unitarily isomorphic to a unitary Virasoro $\operatorname{VOA} L(c, 0)$ for some allowed value of $c$ as in Example 2.5.

\section{B. Rank-one lattice type VOAs}

We recall from Ref. 17, Sec. 2; Ref. 22, Sec. 4; Ref. 33, Sec. 7.1; Ref. 43, Sec. 5.4; and Ref. 48, Chap. 6 some facts about rank-one lattice type VOAs. Let $L$ be an even rank-one positive definite lattice, and let $(\cdot \mid \cdot)$ be the corresponding nondegenerate symmetric bilinear form. We set $\mathfrak{h}:=\mathbb{C} \otimes_{\mathbb{Z}} L$ and indicate the extension of $(\cdot \mid \cdot)$ by bilinearity to $\mathfrak{h}$ with the same symbol. Then, there exist $N \in \mathbb{Z}_{>0}$ and $J \in \mathfrak{h}$, which we call current vector, such that $(J \mid J)=1$ and $L \cong L_{2 N}:=\mathbb{Z} \sqrt{2 N} J$. Let

$$
\widehat{\mathfrak{h}}:=\mathfrak{h} \otimes \mathbb{C}\left[t, t^{-1}\right] \oplus \mathbb{C} K
$$

be the affine Lie algebra with generators $J_{n}:=J \otimes t^{n}$, for $n \in \mathbb{Z}$, and central element $K$, with the commutation relations

$$
\left[J_{m}, J_{n}\right]=m \delta_{m,-n} K
$$

$\widehat{\mathfrak{h}}$ is also called the Heisenberg or oscillator algebra. For $\alpha \in \mathfrak{h}$ and $n \in \mathbb{Z}$, we write $\alpha_{n}:=\alpha \otimes t^{n} \in \widehat{\mathfrak{h}}$. Let $U(\widehat{\mathfrak{h}})$ be the universal enveloping algebra of $\widehat{\mathfrak{h}}$ (see Ref. 40, Chap. V). A linear basis for $U(\widehat{\mathfrak{h}})$ is given by

$$
J_{n_{1}} \cdots J_{n_{s}} K^{k}
$$

for $s, k \in \mathbb{Z}_{\geq 0}, n_{i} \in \mathbb{Z}$ such that $n_{i} \leq n_{j}$ if $i<j$. We define the irreducible $\widehat{\mathfrak{h}}$-module

$$
M(1):=U(\widehat{\mathfrak{h}}) / \mathcal{I}
$$

where $\mathcal{I}$ is the ideal in $U(\widehat{\mathfrak{h}})$ generated by

$$
\left\{(K-1), J_{n}: n \in \mathbb{Z}_{\geq 0}\right\} .
$$

Then, from (4), we deduce that $M(1)$ is linearly generated by the following classes of elements:

$$
[1]=\left[K^{k}\right],\left[J_{n_{1}} \cdots J_{n_{s}}\right]=\left[J_{n_{1}} \cdots J_{n_{s}} K^{k}\right], \quad n_{1} \leq \cdots \leq n_{s}<0, k, s \in \mathbb{Z}_{\geq 0},
$$


which we simply indicate with

$$
\Omega, J_{n_{1}} \cdots J_{n_{s}} \Omega,
$$

respectively.

Define the vector space

$$
V_{L_{2 N}}:=M(1) \otimes_{\mathbb{C}} \mathbb{C}\left[L_{2 N}\right],
$$

where $\mathbb{C}\left[L_{2 N}\right]$ is the group algebra, which we describe as linearly generated by formal elements $e^{\alpha}$, for $\alpha \in L_{2 N}$ and multiplication given by

$$
e^{\alpha} e^{\beta}=e^{\alpha+\beta}
$$

In particular, $1:=e^{0}$ is the identity. Note that a linear set of generators for $V_{L_{2 N}}$ is given by

$$
\left\{v \otimes e^{\alpha} \mid v \in M(1), \alpha \in L_{2 N}\right\} .
$$

Note also that for $v$ as in (5), (7) is a linear basis for $V_{L_{2 N}}$.

Thus, $V_{L_{2 N}}$ has a VOA structure, which we sum up in the following theorem. For the proof, see Ref. 43, Theorem 5.5, Proposition 5.5 and Ref. 48, Theorem 6.5.3. Note that in the above references, the construction of the VOA $V_{L}$ associated with an even lattice $L$ involves the twisted group algebra $\mathbb{C}_{\epsilon}[L]$ instead of the group algebra defined above. In $\mathbb{C}_{\epsilon}[L]$, the multiplication (6) is twisted by a 2-cocycle $\epsilon$. In any case, it turns out that the resulting VOA structure is independent of the choice of the 2-cocycle $\epsilon$ for the twisting. Thus, we are allowed to use the trivial 2-cocycle, which is an admissible choice for the case of even rank-one lattices $L_{2 N}$.

Theorem 2.7. For all $N \in \mathbb{Z}_{>0}, V_{L_{2 N}}$ has a structure of simple VOA of CFT type with vacuum vector $\Omega \otimes 1$ and the following data:

(i) The state-field correspondence is given by

$$
\begin{aligned}
Y\left(\alpha_{-1} \Omega \otimes 1, z\right) & =\alpha(z):=\sum_{j \in \mathbb{Z}} \alpha_{j} z^{-j-1} \\
Y\left(\Omega \otimes e^{\alpha}, z\right) & =e_{\alpha} z^{\alpha_{0}} E_{+}(\alpha, z) E_{-}(\alpha, z), \\
E_{+}(\alpha, z) & :=\exp \left(-\sum_{j<0} \frac{\alpha_{j}}{j} z^{-j}\right) \\
E_{-}(\alpha, z) & :=\exp \left(-\sum_{j>0} \frac{\alpha_{j}}{j} z^{-j}\right) \\
Y\left(J_{n_{1}} \cdots J_{n_{s}} \Omega \otimes e^{\alpha}, z\right) & =: \prod_{j=1}^{s}\left(\partial^{\left(-n_{j}-1\right)} J(z)\right) Y\left(\Omega \otimes e^{\alpha}, z\right):
\end{aligned}
$$

where $\alpha \in \mathfrak{h}$ in (8), whereas $\alpha \in L_{2 N}$ in (9)-(12). In (12), : : : indicates the normally ordered product of fields. In formulas (8)-(11), $e_{\alpha}$ is the operator on $V_{L_{2 N}}$ of left multiplication by $\Omega \otimes e^{\alpha}$, whereas operators $\alpha_{j}$ and $z^{\alpha_{0}}$ act on $v \otimes e^{\beta} \in V_{L_{2 N}}$ in the following manner:

$$
\begin{aligned}
\alpha_{0}\left(v \otimes e^{\beta}\right) & =(\alpha \mid \beta) v \otimes e^{\beta}, \\
\alpha_{j}\left(v \otimes e^{\beta}\right) & =\left(\alpha_{j} v\right) \otimes e^{\beta} \quad \forall j \in \mathbb{Z} \backslash\{0\}, \\
z^{\alpha_{0}}\left(v \otimes e^{\beta}\right) & =z^{(\alpha \mid \beta)} v \otimes e^{\beta} .
\end{aligned}
$$

(ii) The conformal vector with central charge 1 of $V_{L_{2 N}}$ is

$$
v \otimes 1:=\frac{1}{2}\left(J_{-1} J_{-1} \Omega \otimes 1\right)
$$

Thus, the corresponding Virasoro subalgebra is $L(1,0)$. The energy-momentum field

$$
Y(v \otimes 1, z)=\sum_{m \in \mathbb{Z}} L_{m} z^{-m-2}
$$

is defined through formulas 


$$
\begin{aligned}
L_{0}\left(J_{n_{1}} \cdots J_{n_{s}} \Omega \otimes e^{\alpha}\right) & =\left(\frac{(\alpha \mid \alpha)}{2}-\sum_{j=1}^{s} n_{j}\right) J_{n_{1}} \cdots J_{n_{s}} \Omega \otimes e^{\alpha}, \\
L_{m} & =\frac{1}{2} \sum_{j \in \mathbb{Z}} J_{j} J_{m-j}, \quad \forall m \in \mathbb{Z} \backslash\{0\},
\end{aligned}
$$

and it satisfies the commutation relations

$$
\begin{aligned}
{\left[L_{m}, J_{n}\right] } & =-n J_{m+n} \quad \forall m, n \in \mathbb{Z}, \\
{\left[L_{m}, L_{n}\right] } & =(m-n) L_{m+n}+\frac{m^{3}-m}{12} \delta_{m,-n} \quad \forall m, n \in \mathbb{Z} .
\end{aligned}
$$

In particular, $L_{-2}(\Omega \otimes 1)=v \otimes 1$. Furthermore, the vectors $J_{-1} \Omega \otimes 1$ and $\Omega \otimes e^{\alpha}$ are primary of conformal weights 1 and $\frac{(\alpha \mid \alpha)}{2}$, respectively.

From Ref. 33, (7.1.42), (3.2.17), and (2.1.11), we have that

$$
\begin{gathered}
E_{+}(\alpha, z)=I+\sum_{n=1}^{+\infty} \frac{\left(-\sum_{j<0} \frac{\alpha_{j}}{j} z^{-j}\right)^{n}}{n !}, \\
\left(-\sum_{j<0} \frac{\alpha_{j}}{j} z^{-j}\right)^{n}=\sum_{m=n}^{\infty}\left(\sum_{\substack{j_{1}+\cdots+j_{j}=m \\
j_{k}>0}} \frac{\alpha_{-j_{1} \cdots \alpha_{-j_{n}}}}{j_{1} \cdots j_{n}}\right) z^{m}, \\
E_{-}(\alpha, z)=I+\sum_{n=1}^{+\infty} \frac{\left(-\sum_{j>0} \frac{\alpha_{j}}{j} z^{-j}\right)^{n}}{n !}, \\
\left(-\sum_{j>0} \frac{\alpha_{j}}{j} z^{-j}\right)^{n}=(-1)^{n} \sum_{m=n}^{\infty}\left(\sum_{j_{1}+\cdots+j_{n}=m} \frac{\alpha_{j_{1} \cdots \alpha_{j_{n}}}}{j_{1} \cdots j_{n}}\right) z^{-m} .
\end{gathered}
$$

Remark 2.8. Note that the operators $e_{\alpha}$ and $z^{\alpha_{0}}$ commute with $E_{ \pm}(\alpha, z)$ (see also Ref. 48, Proposition 6.4.5).

From Eqs. (13), (14), (17), and (18), we obtain

$$
\begin{aligned}
& L_{0}(v \otimes 1)=\left(\sum_{j \in \mathbb{Z}_{>0}} J_{-j} J_{j} v\right) \otimes 1, \\
& L_{m}(v \otimes 1)=\left(\frac{1}{2} \sum_{j \in \mathbb{Z}} J_{j} J_{m-j} v\right) \otimes 1, \quad \forall m \in \mathbb{Z} \backslash\{0\}
\end{aligned}
$$

for all $v \in M(1)$, so we write with an abuse of notation $\left(L_{m} v\right) \otimes 1$ or just $L_{m} v$ instead of $L_{m}(v \otimes 1)$ for all $m \in \mathbb{Z}$.

Define an antilinear involution $\theta$ on $V_{L_{2 N}}$ which acts in the following manner on basis elements:

$$
J_{n_{1}} \cdots J_{n_{s}} \Omega \otimes e^{\alpha} \longmapsto(-1)^{s} J_{n_{1}} \cdots J_{n_{s}} \Omega \otimes e^{-\alpha} .
$$

By Ref. 22, Theorem 4.12, $V_{L_{2 N}}$ has a structure of unitary VOA with PCT operator $\theta$. Note also that all unitary structures on a simple VOA are equivalent up to unitary isomorphism (see Ref. 10, p. 38).

In the following, we list some well-known unitary subalgebras of $V_{L_{2 N}}$ and we describe actions of some unitary automorphisms of $V_{L_{2 N}}$ and the respective fixed point subalgebras.

A unitary subalgebra of $V_{L_{2 N}}$ is $M(1) \otimes_{\mathbb{C}} \mathbb{C} 1$ [which we indicate just with $M(1)$ ] with vacuum vector $\Omega \otimes 1$, conformal vector $v \otimes 1$, and PCT operator $\left.\theta\right|_{M(1)}$. More in general, this is a well-known unitary VOA with central charge 1, called the Heisenberg or current VOA (see Ref. 22, Sec. 4.3). 
Let $\phi$ be the linear automorphism on $V_{L_{2 N}}$ defined by the following action on the basis elements:

$$
J_{n_{1}} \cdots J_{n_{s}} \Omega \otimes e^{\alpha} \longmapsto(-1)^{s} J_{n_{1}} \cdots J_{n_{s}} \Omega \otimes e^{-\alpha}
$$

[not to be confused with the antilinear PCT operator $\theta$ in (27)]. Note that $\phi$ restricts to a linear automorphism on $M(1)$. Then, we define the orbifold subalgebras $V_{L_{2 N}}^{+}$and $M(1)^{+}$as fixed points of $V_{L_{2 N}}$ and $M(1)$, respectively, with respect to $\phi$ (see also Ref. 17, Sec. 2).

We identify every $t \in \mathbb{T}:=\mathbb{R} / 2 \pi \mathbb{Z}$ with its representative in $[0,2 \pi)$ and for any positive integer $N$, we define an action on $V_{L_{2 N}}$ by automorphisms (see Ref. 25, Sec. 2.3)

$$
g_{2 N, t}:=\exp \left(i \frac{t}{\sqrt{2 N}} J_{0}\right)=\sum_{n \geq 0} \frac{\left(i \frac{t}{\sqrt{2 N}} J_{0}\right)^{n}}{n !}
$$

which act on generators of $V_{L_{2 N}}$ in the following manner:

$$
v \otimes e^{\alpha} \longmapsto e^{i \frac{t}{\sqrt{2 N}}(J \mid \alpha)} v \otimes e^{\alpha} .
$$

Note that $J_{0}$ leaves invariant the finite-dimensional eigenspaces of $L_{0}$ so that $\exp \left(i \frac{t}{\sqrt{2 N}} J_{0}\right)$ is a well-defined vector space endomorphism of $V_{L_{2 N}}$. Note also that for $\alpha=\sqrt{2 N} J \in L_{2 N}$, we have $e^{i \frac{t}{\sqrt{2 N}}(J \mid \alpha)}=e^{i t}$ so that $g_{2 N, t}$ is not the identity when $t \neq 0, t \in[0,2 \pi)$. We include the following well-known facts with proofs for the reader's convenience.

Proposition 2.9. For any positive integer $N$, the automorphisms $g_{2 N, t}$ and $\phi$ defined above give an embedding of $D_{\infty}=\mathbb{T} \rtimes \mathbb{Z} 2$ into $\operatorname{Aut}_{(\cdot \mid)} V_{L_{2 N}}$. Moreover,

$$
\begin{aligned}
V_{L_{2 N}}^{\mathbb{T}} & =M(1), \\
V_{L_{2 N}}^{D_{\infty}} & =M(1)^{+} .
\end{aligned}
$$

Proof. For every fixed $N$, let $G_{2 N} \cong \mathbb{T}$ and $H \cong \mathbb{Z}_{2}$ be the groups generated by the automorphisms $g_{2 N, t}$ for all $t \in \mathbb{T}$ and $\phi$, respectively. Using (28) and (30), it is easy to verify that $\phi g_{2 N, t} \phi=g_{2 N, t}^{-1}$ for all $g_{2 N, t} \in G_{2 N}$. It follows that the group generated by $G_{2 N}$ and $H$ is isomorphic to $D_{\infty}$.

Now, we prove that the automorphisms $g_{2 N, t}$ and $\phi$ are unitary. Recall from Ref. 10, Remark 5.18 that if $V$ is a simple unitary VOA then an automorphism $g$ of $V$ is unitary if and only if $g$ commutes with the PCT operator. The above condition is easily verified on basis elements of $V_{L_{2 N}}$. Thus, $D_{\infty}$ embeds into Aut $(\cdot \mid \cdot) V_{L_{2 N}}$.

Note that (32) follows from (31) by definition. To prove (31), consider $a \in V_{L_{2 N}}^{\mathbb{T}}$ and its linear decomposition into basis elements, that is,

$$
a=\sum_{j=1}^{M} a_{j} v_{j} \otimes e^{\alpha_{j}},
$$

with $\mathbb{C} \ni a_{j} \neq 0$ for all $j$ and $v_{j}$ as one of the vectors in (5). Applying a generic automorphism $g_{2 N, t}$ to both sides of (33), we obtain

$$
\sum_{j=1}^{M} a_{j} v_{j} \otimes e^{\alpha_{j}}=a=g_{2 N, t}(a)=\sum_{j=1}^{M} e^{i \frac{t}{\sqrt{2 N}}\left(J \mid \alpha_{j}\right)} a_{j} v_{j} \otimes e^{\alpha_{j}} .
$$

By linear independence of $v_{j} \otimes e^{\alpha_{j}},(34)$ is satisfied if and only if $e^{i \frac{t}{\sqrt{2 N}}\left(J \mid \alpha_{j}\right)}=1$ for all $j$ and $t \in \mathbb{T}$, i.e., $\alpha_{j}=0$ for all $j$. To sum up, $a \in V_{L_{2 N}}^{\mathbb{T}}$ if and only if $\alpha_{j}=0$ for all $j$, which is equivalent to $a \in M(1)$. This completes the proof.

In the following, for every fixed $N$, we identify $\mathbb{T}$ with the closed subgroup $G_{2 N}$ of Aut $(\cdot) V_{L_{2 N}}$ as in the Proof of Proposition 2.9. We also identify $\mathbb{Z}_{k} \subset \mathbb{T}$ with the cyclic subgroup of $A_{u t}(\cdot \cdot) V_{L_{2 N}}$ of order $k$ generated by automorphisms $g_{N}, \frac{2 m \pi}{k}$ for $m \in\{0, \ldots, k-1\}$ and $D_{k}:=\mathbb{Z}_{k} \rtimes \mathbb{Z}_{2}$ with the corresponding dihedral subgroup of $\operatorname{Aut}_{(\cdot \cdot)} V_{L_{2 N}}$ of order $k$.

Note that $L_{2 N k^{2}}$ is a sublattice of $L_{2 N}$ for all positive integers $N$ and $k$. This inclusion induces an isomorphism of vector spaces

$$
\iota: V_{L_{2 N k^{2}}} \longleftrightarrow V:=\bigoplus_{\alpha \in L_{2 N k^{2}}} M(1) \otimes_{\mathbb{C}} \mathbb{C} e^{\alpha} \subset V_{L_{2 N}}
$$

$V$ is clearly a vertex subalgebra of $V_{L_{2 N}}$, thanks to Theorem 2.7. Note that $v \in V$ and that the PCT operator $\theta$ is independent of $N$ and $k$. Thus, $V$ is a unitary subalgebra of $V_{L_{2 N}}$ as in Definition 2.1. It follows that (35) is a unitary isomorphism of unitary VOAs. In particular, $\iota(M(1))=M(1)$. Therefore, from now onwards, we consider $V_{L_{2 N k^{2}}}$ as a unitary subalgebra of $V_{L_{2 N}}$. 
Proposition 2.10. For every positive integer $N$ and $k$, we have that

$$
\begin{aligned}
V_{L_{2 N}}^{Z_{k}} & =V_{L_{2 N k^{2}}}, \\
V_{L_{2 N}}^{D_{k}} & =V_{L_{2 N k^{2}}^{+}} .
\end{aligned}
$$

Proof. First, note that (37) follows from (36) by definition. Then, it is sufficient to prove that $V_{L_{2 N}}^{\mathbb{Z}_{k}}$ is equal to $V$ as in (35) to conclude. Using the same argument as in the Proof of Proposition 2.9, we have that $a \in V_{L_{2 N}}^{\mathbb{Z}_{k}}$ if and only if $e^{i \frac{2 \pi}{k \sqrt{2 N}}\left(J \mid \alpha_{j}\right)}=1$ for all $j . \alpha_{j} \in \mathbb{Z} \sqrt{2 N} J$ for all $j$; thus, the former condition is satisfied if and only if $\alpha_{j} \in \mathbb{Z} \sqrt{2 N} k J$ for all $j$. Then, $V=V_{L_{2 N}}^{\mathbb{Z}_{k}}$ as desired.

Remark 2.11. The closed subgroups of $D_{\infty} \subset \operatorname{Aut}_{(\cdot \cdot)} V_{L_{2 N}}$ are the circle group $\mathbb{T}$, cyclic groups $\mathbb{Z}_{k}$, dihedral groups $D_{k}$, and their conjugates $D_{k}^{t}:=g_{2 N, t} D_{k} g_{2 N,-t}$ for all $t \in \mathbb{T}$. Note that $D_{k}^{t}=D_{k}^{t+\pi}$ for all $k \in \mathbb{Z}_{>0}$ and $t \in \mathbb{T}\left(g_{2 N, \pi}\right.$ is in the center of $\left.D_{\infty}\right)$. Furthermore, obviously $D_{k}=D_{k}^{t}$ for all $k \in \mathbb{Z}_{>0}$ and $t \in \mathbb{Z}_{k}$. Thus, it is easy to verify that $V_{L_{2 N}}^{D_{k}^{t}}=g_{2 N, t}\left(V_{L_{2 N}}^{D_{k}}\right)$ for all $k \in \mathbb{Z}_{>0}$ and $t \in \mathbb{T}$, which is unitarily isomorphic to $V_{L_{2 N}}^{D_{k}}$ for all $k \in \mathbb{Z}_{>0}$ and $t \in \mathbb{T}$.

The VOAs just mentioned above have a decomposition into Virasoro modules, as described in Ref. 17, Sec. 2. In particular, if $N$ is not a perfect square (the square of a positive integer), then we have the following decompositions into irreducible Virasoro modules:

$$
\begin{aligned}
V_{L_{2 N}} & =\bigoplus_{p \geq 0} L\left(1, p^{2}\right) \oplus \bigoplus_{m>0} 2 L\left(1, N m^{2}\right), \\
M(1) & =\bigoplus_{p \geq 0} L\left(1, p^{2}\right), \\
V_{L_{2 N}}^{+} & =\bigoplus_{p \geq 0} L\left(1,4 p^{2}\right) \oplus \bigoplus_{m>0} L\left(1, N m^{2}\right), \\
M(1)^{+} & =\bigoplus_{p \geq 0} L\left(1,4 p^{2}\right) .
\end{aligned}
$$

Remark 2.12. As stated in Ref. 17, Sec. 2, the decompositions in (38) and (39) are due to the fact that the following isomorphisms of Virasoro modules hold:

$$
M(1) \otimes_{\mathbb{C}} \mathbb{C} e^{ \pm \sqrt{2 N} m J} \cong L\left(1, N m^{2}\right), \quad \forall m \in \mathbb{Z}_{>0} .
$$

Thus, for all $m>0, \Omega \otimes e^{ \pm \sqrt{2 N} m J}$ form a basis for the subspace of $V_{L_{2 N}}$ of primary vectors of conformal weight $N m^{2}$. As a matter of fact, it is straightforward to check that the former elements are homogeneous of conformal weight $\mathrm{Nm}^{2}$ for all $m>0$ by (17) and that $L_{n}\left(\Omega \otimes e^{ \pm \sqrt{2 N} m J}\right)=0$ for all $n>0$ and $m>0$ by (18). Thus, they generate (as Virasoro modules) the two copies of $L\left(1, N m^{2}\right)$. This also implies that every $L\left(1, N m^{2}\right)$ in (40) is generated by the $\phi$-invariant primary vector $\Omega \otimes\left(e^{\sqrt{2 N} m J}+e^{-\sqrt{2 N} m J}\right)$ of conformal weight $N m^{2}$.

\section{THE CLASSIFICATION IN THE VOA SETTING}

In this section, we classify all unitary subalgebra of $V_{L_{2 N}}$, for all $N \in \mathbb{Z}_{>0}$. To do this, we state the following Galois correspondence for unitary VOAs which is crucial for the classification result. This is a variant of Ref. 10, Theorem 7.7 which can be entirely formulated and proved in the unitary VOA setting, thanks to Ref. 24, Theorem 3.

Theorem 3.1. Let $V$ be a simple unitary VOA and $G$ a closed subgroup of $\operatorname{Aut}_{(\cdot \mid \cdot)} V$ which is topologically isomorphic to a Lie group. Then, the map $H \mapsto V^{H}$ gives a one-to-one correspondence between the closed subgroups $H$ of $G$ and the unitary subalgebras $W \subset V$ containing $V^{G}$.

Proof. We first show that every unitary subalgebra $W \subset V$ which contains $V^{G}$ is an orthogonally complemented subVOA in the sense of Ref. 24, Definition 2. To this end, it is rather straightforward to see that in our case the subspace in Eq. (1.3) in Ref. 24, Definition 2 coincides with the orthogonal complement

$$
W^{\perp}:=\{a \in V \mid(a \mid b)=0 \text { for all } b \in W\}
$$


which is easily seen to be a $W$-submodule of $V$ because of the invariance property of the scalar product and the fact that $W$ is a unitary subalgebra. Now, for every closed subgroup $H$ of $G, V^{H}$ is a unitary subalgebra of $V$ by Example 2.4. Moreover, since Aut $(\cdot \cdot \cdot) V$ is compact by Ref. 10, Lemma 5.20, G must be topologically isomorphic to a compact Lie group. Then, the result follows from Ref. 24 , Theorem 3.

The unitary subalgebras of $V_{L_{2}}$ have already been classified in Ref. 10, Theorem 8.13. Here, we give a proof in the unitary VOA setting using the Galois correspondence stated in Theorem 3.1 instead of Ref. 10, Theorem 7.7.

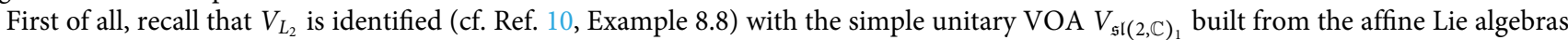
$\mathfrak{s l}(2, \mathbb{C})_{1}$ associated with the Lie group SU(2) (see Ref. 42, Chap. 7, Refs. 36 and 52). Consider the orthonormal generators $J^{a}$ for $a \in\{x, y, z\}$ of the complex Lie algebra $\mathfrak{s l}(2, \mathbb{C})$ and corresponding elements $J_{0}^{a}$ of the associated loop algebra. Then, we have a group of unitary isomorphisms of $V_{\mathfrak{s l}(2, \mathbb{C})_{1}}$, isomorphic to $\mathrm{SO}(3)$ and generated by operators of the form $\exp \left(i t j_{0}^{a}\right)$ for all $t \in \mathbb{T}$ and all $a \in\{x, y, z\}$. Then, $\mathrm{SO}(3) \subseteq \mathrm{Aut}_{(\cdot \mid \cdot)} V_{L_{2}}$ (see Ref. 10, p. 63). Remember that the closed subgroups of $\mathrm{SO}(3)$ are, up to isomorphism, the circle group $\mathbb{T}$, all cyclic groups $\mathbb{Z}_{k}$, the infinite dihedral group $D_{\infty}$, all finite dihedral groups $D_{k}$, and the three platonic groups $E_{m}$. Finally, note that the action of $D_{\infty}$ introduced in Proposition 2.9 can be embedded in $\mathrm{SO}(3)$ as described above, identifying the current vector $J$ with $\sqrt{2} J^{z}$, cf. Ref. 2, Sec. 5B. Therefore, we have the following.

Theorem 3.2. All unitary subalgebras of $V_{L_{2}} \cong V_{\mathfrak{s l}(2, \mathbb{C})_{1}}$ are given by the family of fixed point subalgebras $V_{L_{2}}^{H}$ for every closed subgroup $H \subseteq \mathrm{SO}(3)$ together with the trivial subalgebra $\mathbb{C} \Omega \otimes 1$. In particular, $V_{L_{2}}^{\mathrm{SO}(3)}=L(1,0)$ and consequently $\operatorname{Aut}_{(\cdot \cdot)} V_{L_{2}}=\mathrm{SO}(3)$.

Proof. Let $W$ be a nontrivial subalgebra. Then, by Ref. 10, Lemma 8.10, $L(1,0) \subset W$. Moreover, by Ref. 10, Proposition 8.11 (cf. also Ref. 17, Corollary 2.4), $L(1,0)=V_{L_{2}}^{S O(3)}$ and the claim follows from Theorem 3.1.

For references about VOAs built from affine Lie algebras, cf. Ref. 43, Example 4.9a and Sec. 5.7 and Ref. 48, Sec. 6.2; Ref. 33, Sec. 7.2; and Ref. 22, Sec. 4.2 for the unitary structure.

For $N$ different from a perfect square, the classification of unitary subalgebras of $V_{L_{2 N}}$ will rely on Theorem 3.8, the key ingredient of our classification result, which states that any unitary subalgebra properly containing $L(1,0)$ also contains $M(1)^{+}$. To prove it, we begin with some preliminary results.

Lemma 3.3. Let $N \in \mathbb{Z}_{>0}$.

(i) The vector

$$
u \otimes 1:=\left(\frac{1}{2}\left(J_{-1}\right)^{4} \Omega-J_{-3} J_{-1} \Omega+\frac{3}{4}\left(J_{-2}\right)^{2} \Omega\right) \otimes 1
$$

is primary of conformal weight 4. In particular, if $N$ is not a perfect square, then it is the highest weight vector of the unique irreducible Virasoro submodule $L(1,4)$ of $V_{L_{2 N}}$ as in $(38)$.

(ii) We also have

$$
\begin{aligned}
L_{-2}(v \otimes 1) & =\left(\frac{1}{4}\left(J_{-1}\right)^{4} \Omega+J_{-3} J_{-1} \Omega\right) \otimes 1, \\
L_{-4}(\Omega \otimes 1) & =\left(\frac{1}{2}\left(J_{-2}\right)^{2} \Omega+J_{-3} J_{-1} \Omega\right) \otimes 1 .
\end{aligned}
$$

Proof. (i) From formula (17), it is clear that $u \otimes 1$ is homogeneous of conformal weight 4 . According to (38), if $u \otimes 1$ is primary and $N$ is not a perfect square, it will be the highest weight vector of the unique irreducible Virasoro submodules $L(1,4)$ of $V_{L_{2 N}}$. We need to prove that $L_{m} u=0$ for all $m \geq 1$. Using Eq. (19), we see that

$$
\begin{aligned}
\frac{1}{2} L_{m}\left(J_{-1}\right)^{4} \Omega= & \frac{1}{2}\left(J_{m-1}\left(J_{-1}\right)^{3} \Omega+J_{-1} J_{m-1}\left(J_{-1}\right)^{2} \Omega\right. \\
& \left.+\left(J_{-1}\right)^{2} J_{m-1} J_{-1} \Omega+\left(J_{-1}\right)^{3} J_{m-1} \Omega+\left(J_{-1}\right)^{4} L_{m} \Omega\right) \\
-L_{m} J_{-3} J_{-1} \Omega= & -\left(3 J_{m-3} J_{-1} \Omega+J_{-3} J_{m-1} \Omega+J_{-3} J_{-1} L_{m} \Omega\right), \\
\frac{3}{4} L_{m}\left(J_{-2}\right)^{2} \Omega= & \frac{3}{4}\left(2 J_{m-2} J_{-2} \Omega+2 J_{-2} J_{m-2} \Omega+\left(J_{-2}\right)^{2} L_{m} \Omega\right) .
\end{aligned}
$$

Note that the last term on the right-hand side of each of Eqs. (43)-(45) is 0 because $L_{m} \Omega=0$ for all $m \geq 1$. Moreover, by the commutation relations (3), we know $J_{j}$ and $J_{k}$ commute if $k \neq-j$. This means that for all $m \geq 5$, every term in Eqs. (43)-(45) is zero since $J_{j} \Omega=0$ for all $j \geq 0$. Therefore, it remains to study the four cases $m \in\{1,2,3,4\}$ one by one. For $m=1$, we have 


$$
\begin{aligned}
& \frac{1}{2} L_{1}\left(J_{-1}\right)^{4} \Omega=2\left(J_{-1}\right)^{3} J_{0} \Omega=0 \\
& -L_{1} J_{-3} J_{-1} \Omega=-3 J_{-2} J_{-1} \Omega-J_{-3} J_{0} \Omega=-3 J_{-2} J_{-1} \Omega \\
& \frac{3}{4} L_{1}\left(J_{-2}\right)^{2} \Omega=\frac{3}{4}\left(2 J_{-1} J_{-2} \Omega+2 J_{-2} J_{-1} \Omega\right)=3 J_{-2} J_{-1} \Omega,
\end{aligned}
$$

and the sum is clearly 0 . For $m=2$,

$$
\begin{aligned}
\frac{1}{2} L_{2}\left(J_{-1}\right)^{4} \Omega= & \frac{1}{2}\left(J_{1}\left(J_{-1}\right)^{3} \Omega+J_{-1} J_{1}\left(J_{-1}\right)^{2} \Omega+\right. \\
& \left.+\left(J_{-1}\right)^{2} J_{1} J_{-1} \Omega+\left(J_{-1}\right)^{3} J_{1} \Omega\right) \\
= & \frac{1}{2}\left((3+2+1)\left(J_{-1}\right)^{2} \Omega+4\left(J_{-1}\right)^{3} J_{1} \Omega\right) \\
= & 3\left(J_{-1}\right)^{2} \Omega \\
-L_{2} J_{-3} J_{-1} \Omega= & -3 J_{-1} J_{-1} \Omega-J_{-3} J_{1} \Omega=-3\left(J_{-1}\right)^{2} \Omega \\
\frac{3}{4} L_{2}\left(J_{-2}\right)^{2} \Omega= & \frac{3}{4}\left(2 J_{0} J_{-2} \Omega+2 J_{-2} J_{0} \Omega\right)=0,
\end{aligned}
$$

and again, the sum is 0 . For $m=3$,

$$
\begin{aligned}
& \frac{1}{2} L_{3}\left(J_{-1}\right)^{4} \Omega=2\left(J_{-1}\right)^{3} J_{2} \Omega=0, \\
& -L_{3} J_{-3} J_{-1} \Omega=-3 J_{0} J_{-1} \Omega-J_{-3} J_{2} \Omega=0, \\
& \frac{3}{4} L_{3}\left(J_{-2}\right)^{2} \Omega=\frac{3}{4}\left(2 J_{1} J_{-2} \Omega+2 J_{-2} J_{1} \Omega\right)=0 .
\end{aligned}
$$

Finally, for $m=4$,

$$
\begin{aligned}
& \frac{1}{2} L_{4}\left(J_{-1}\right)^{4} \Omega=2\left(J_{-1}\right)^{3} J_{3} \Omega=0 \\
& -L_{4} J_{-3} J_{-1} \Omega=-3 J_{1} J_{-1} \Omega-J_{-3} J_{3} \Omega=-3 \Omega, \\
& \frac{3}{4} L_{4}\left(J_{-2}\right)^{2} \Omega=\frac{3}{4}\left(2 J_{2} J_{-2} \Omega+2 J_{-2} J_{2} \Omega\right)=3 \Omega,
\end{aligned}
$$

which adds up to 0 as well.

(ii) From (ii) of Theorem 2.7, we have that

$$
\begin{aligned}
L_{-2} v=\frac{1}{2} L_{-2}\left(J_{-1}\right)^{2} \Omega & =\frac{1}{2}\left(2 J_{-3} J_{-1} \Omega+\left(J_{-1}\right)^{2} L_{-2} \Omega\right) \\
& =J_{-3} J_{-1} \Omega+\frac{1}{4}\left(J_{-1}\right)^{4} \Omega,
\end{aligned}
$$

and using (18),

$$
\begin{aligned}
L_{-4} \Omega=\frac{1}{2} \sum_{j \in \mathbb{Z}} J_{j} J_{-4-j} \Omega=\frac{1}{2} \sum_{j=-3}^{-1} J_{j} J_{-4-j} \Omega & =\frac{1}{2}\left(2 J_{-3} J_{-1} \Omega+\left(J_{-2}\right)^{2} \Omega\right) \\
& =J_{-3} J_{-1} \Omega+\frac{1}{2}\left(J_{-2}\right)^{2} \Omega .
\end{aligned}
$$

Proposition 3.4. Let $N, m$ be positive integers such that $g:=\sqrt{2 N} m \geq 2$. Let $b \in \mathbb{C} \backslash\{0\}$ and set

$$
e_{b}^{g}:=\Omega \otimes\left(e^{g J}+b e^{-g J}\right)
$$


Then,

$$
\begin{aligned}
\left(\Omega \otimes e^{ \pm g J}\right)_{\left(g^{2}-2\right)}\left(\Omega \otimes e^{\mp g J}\right) & =\left( \pm g J_{-1} \Omega\right) \otimes 1 \\
\left(e_{b}^{g}\right)_{\left(g^{2}-5\right)} e_{b}^{g} & =b v_{g} \otimes 1,
\end{aligned}
$$

where

$$
v_{g}:=\frac{g^{4}}{12}\left(J_{-1}\right)^{4} \Omega+\frac{2 g^{2}}{3} J_{-3} J_{-1} \Omega+\frac{g^{2}}{4}\left(J_{-2}\right)^{2} \Omega
$$

Proof. Note that the $z^{-g^{2}+4}$-coefficient of the formal $z$-series $Y\left(e_{b}^{g}, z\right) e_{b}^{g}$ corresponds to $\left(e_{b}^{g}\right)_{\left(g^{2}-5\right)} e_{b}^{g}$. We have that

$$
\begin{aligned}
Y\left(e_{b}^{g}, z\right) e_{b}^{g} & =A+b B \\
A & :=Y\left(\Omega \otimes e^{g J}, z\right)\left(\Omega \otimes e^{g J}\right)+b^{2} Y\left(\Omega \otimes e^{-g J}, z\right)\left(\Omega \otimes e^{-g J}\right) \\
B & :=Y\left(\Omega \otimes e^{g J}, z\right)\left(\Omega \otimes e^{-g J}\right)+Y\left(\Omega \otimes e^{-g J}, z\right)\left(\Omega \otimes e^{g J}\right) .
\end{aligned}
$$

From formulas (23) and (24), we deduce that $E_{-}(c g J, z)\left(\Omega \otimes e^{d g J}\right)$ is equal to

$$
\left(\Omega+\sum_{n=1}^{+\infty} \frac{(-1)^{n}}{n !}\left(\sum_{m=n}^{\infty}\left(\sum_{j_{1}+\cdots+j_{n}=m} \frac{(c g)^{n} j_{j_{1}} \cdots j_{j_{n}} \Omega}{j_{1} \cdots j_{n}}\right) z^{-m}\right)\right) \otimes e^{d g J},
$$

for all $c, d \in\{-1,+1\}$. Formula (46) is equal to $\Omega \otimes e^{d g J}$ because $J_{j} \Omega=0$ for all $j \geq 0$ by construction. From Remark 2.8 and (9), we have that

$$
\begin{aligned}
& Y\left(\Omega \otimes e^{ \pm g J}, z\right)\left(\Omega \otimes e^{ \pm g J}\right)=z^{g^{2}}\left(E_{+}( \pm g J, z) \Omega\right) \otimes e^{ \pm 2 g J}, \\
& Y\left(\Omega \otimes e^{ \pm g J}, z\right)\left(\Omega \otimes e^{\mp g J}\right)=z^{-g^{2}}\left(E_{+}( \pm g J, z) \Omega\right) \otimes 1 .
\end{aligned}
$$

From (22), we deduce that the lowest $z$-power in Eq. (47) is $g^{2}>0$. Thus, we can restrict our attention just to $B$ and therefore to Eq. (48) because $-g^{2}+4 \leq 0$. Then, we have to consider the $z^{4}$-coefficient of $E_{+}( \pm g J, z) \Omega$ in Eq. (48). This means that in (21) and (22), we can restrict the calculation to the cases $m=4$ and $n \in\{1,2,3,4\}$. There we find

$$
\begin{array}{ll}
n=1: & \frac{ \pm g}{4} J_{-4}, \\
n=2: & \frac{1}{2}\left(\frac{ \pm g)^{2}}{4}\left(J_{-2}\right)^{2}+\frac{( \pm g)^{2}}{3} J_{-3} J_{-1}+\frac{( \pm g)^{2}}{3} J_{-1} J_{-3}\right), \\
n=3: & \frac{1}{3 !}\left(\frac{( \pm g)^{3}}{2} J_{-2}\left(J_{-1}\right)^{2}+\frac{( \pm g)^{3}}{2} J_{-1} J_{-2} J_{-1}+\frac{( \pm g)^{3}}{2}\left(J_{-1}\right)^{2} J_{-2}\right), \\
n=4: & \frac{1}{4 !}\left(( \pm g)^{4}\left(J_{-1}\right)^{4}\right) .
\end{array}
$$

Using the commutation relations (3), we obtain as $z^{4}$-coefficient of $E_{+}( \pm g J, z) \Omega$ in Eq. (48) the element

$$
\pm \frac{g}{4} J_{-4} \Omega+\frac{g^{2}}{8}\left(J_{-2}\right)^{2} \Omega+\frac{g^{2}}{3} J_{-3} J_{-1} \Omega \pm \frac{g^{3}}{4} J_{-2}\left(J_{-1}\right)^{2} \Omega+\frac{g^{4}}{24}\left(J_{-1}\right)^{4} \Omega .
$$

In order to compute $B$, we have to add up the two versions of (49), so the summands with \pm cancel, while the other ones double, and we obtain for the $z^{-g^{2}+4}$-coefficient of $Y\left(e_{b}^{g}, z\right) e_{b}^{g}$,

$$
b v_{g} \otimes 1=b\left(\frac{g^{4}}{12}\left(J_{-1}\right)^{4} \Omega+\frac{2 g^{2}}{3} J_{-3} J_{-1} \Omega+\frac{g^{2}}{4}\left(J_{-2}\right)^{2} \Omega\right) \otimes 1 .
$$

In the same manner, the $z^{-g^{2}+1}$-coefficient of the formal $z$-series $Y\left(\Omega \otimes e^{ \pm g J}, z\right)\left(\Omega \otimes e^{\mp g J}\right)$ corresponds to $\left(\Omega \otimes e^{ \pm g J}\right)_{\left(g^{2}-2\right)}\left(\Omega \otimes e^{\mp g J}\right)$. Thus, we have to consider the $z$-coefficient of $E_{+}( \pm g$ J,$z) \Omega$ in Eq. (48). This corresponds to fixing $m=1=n$ in formulas (21) and (22) to obtain $\pm g J_{-1}$. Thus, $\left( \pm g J_{-1} \Omega\right) \otimes 1$ are the desired $z^{-g^{2}+1}$-coefficients. 
Proposition 3.5. For every nonzero complex number $g$, the primary vector $u \otimes 1 \in V_{L_{2 N}}$ of conformal weight 4 as in Lemma 3.3 is a linear combination of vectors $L_{-4} \Omega \otimes 1, L_{-2} v \otimes 1$, and $v_{g} \otimes 1$ as in Proposition 3.4.

Proof. It is easy to see that the three vectors form a basis for the vector subspace of $V_{L_{2 N}}$ generated by $\left(J_{-1}\right)^{4} \Omega \otimes 1, J_{-3} J_{-1} \Omega \otimes 1$, and $\left(J_{-2}\right)^{2} \Omega \otimes 1$. It follows that $u \otimes 1$ must be a linear combination of these three vectors.

From previous results, we obtain a generalization of Ref. 17, Theorem 2.9.

Corollary 3.6. For any integer $N$ which is not a perfect square, $V_{L_{2 N}}^{+}$is generated (as VOA) by the conformal vector $v \otimes 1$ and the primary vector $e_{1}^{\sqrt{2 N}}=\Omega \otimes\left(e^{\sqrt{2 N} J}+e^{-\sqrt{2 N} J}\right)$.

Remark 3.7. Note that in Ref. 17, the authors indicate with $\omega$ and $\beta$ our conformal vector $v \otimes 1$ and the current vector $J$, respectively (see Ref. 17, p. 264). Moreover, their $u^{m}$ stands for the highest weight vector of the irreducible Virasoro submodule $L\left(1, m^{2}\right)$ of $M(1)$ (see Ref. 17, p. 268); thus, their $u^{2}$ is our $u \otimes 1$ as in Lemma 3.3. Finally, $e^{n}$ in Ref. 17, p. 269 coincides with our $e_{1}^{\sqrt{2 N}}$, identifying $n$ with $N$ when they are different from a perfect square.

Proof of Corollary 3.6. Due to Remark 2.12, the vector $\Omega \otimes\left(e^{\sqrt{2 N} J}+e^{-\sqrt{2 N} J}\right)$ is in $V_{L_{2 N}}^{+}$. Using Proposition 3.4, the vector $v_{g} \otimes 1 \in V_{L_{2 N}}^{+}$. The result then follows from Proposition 3.5 and Ref. 17, Theorem 2.9.

We are now ready to prove the key ingredient of our classification proof.

Theorem 3.8. Let $N$ be an integer which is not a perfect square. Every unitary subalgebra $W$ of $V_{L_{2 N}}$ properly containing L(1, 0$)$ contains also $M(1)^{+}$.

Proof. Consider the decomposition of $V_{L_{2 N}}$ as in (38). Keeping in mind the notational correspondence given in Remark 3.7, if $W$ contains $L\left(1,4 p^{2}\right)$ for at least one $p>0$, then the theorem follows from Ref. 17, Theorem 2.7(2). Similarly, if $W$ contains $L\left(1, p^{2}\right)$ for at least one odd $p>0$, then we have that $\bigoplus_{j=0}^{2 p} L\left(1, j^{2}\right) \subseteq W$ by Ref. 17, Lemma 2.6. Then, the theorem follows from Ref. 17, Theorem 2.7(2). We therefore have to prove that $W$ contains at least one $L\left(1, p^{2}\right)$ with $p>0$.

Suppose for contradiction that $W$ does not contain any $L\left(1, p^{2}\right)$ with $p>0$, i.e., $W \cap L\left(1, p^{2}\right)=\{0\}$. This implies that $W$ has the following decomposition into Virasoro modules:

where $a_{m} \in\{0,1,2\}$.

$$
W=L(1,0) \oplus \bigoplus_{m>0} a_{m} L\left(1, N m^{2}\right)
$$

We first want to prove that $a_{m} \neq 2$, for all $m$. If $a_{m}$ were equal to 2 for some $m$, then by Remark 2.12, $\Omega \otimes e^{ \pm \sqrt{2 N} m J} \in W$. By Proposition 3.4, $J_{-1} \Omega \otimes 1$ would then lie in $W$, but according to Theorem 2.7(ii), we have $J_{-1} \Omega \otimes 1 \in L(1,1)$, which cannot be the case as $W \cap L(1,1)=\{0\}$ by assumption. Therefore, $a_{m} \in\{0,1\}$; moreover, at least one $a_{m}$ equals 1 because by assumption $W \neq L(1,0)$. Fix such an $m$.

Second, from Remark 2.12, we know that, for this $m$, there exists a primary vector of conformal weight $g^{2} / 2:=N m^{2}$ in $W$ which must be a linear combination of the form $\Omega \otimes\left(a e^{g J}+b e^{-g J}\right)$, for some $a, b \in \mathbb{C}$. $W$ is a unitary subalgebra; thus, by Definition $2.1, W$ must be invariant under the PCT operator $\theta$. If $a$ were 0 , then $\theta\left(\Omega \otimes b e^{-g J}\right)=\Omega \otimes \bar{b} e^{g J}$ would be in $W$, which means that $a_{m}=2$; however, since $a_{m}=1$, we find that $a \neq 0$. Similarly, $b \neq 0$; thus, up to rescaling, we can suppose $a=1$. With $e_{b}^{g}=\Omega \otimes\left(e^{g J}+b e^{-g J}\right) \in W$ as in Proposition 3.4, the vector $v$ $\otimes 1$ must lie in $W$. Furthermore, $L_{-2} v \otimes 1$ and $L_{-4} \Omega \otimes 1$ are in $W$ because they are vectors of $L(1,0)$. Thus, by Proposition $3.5, u \otimes 1$ lies in $W$. On the other hand, $u \otimes 1$ is the primary vector of conformal weight 4 generating $L(1,4)$, so we obtain $W \cap L(1,4) \neq\{0\}$, which leads to a contradiction. Therefore, $W$ must contain at least one $L\left(1, p^{2}\right)$ with $p>0$, which concludes the Proof of the theorem.

Theorem 3.8 also allows us to explicitly calculate $\operatorname{Aut}_{(\cdot) \cdot} V_{L_{2 N}}$ for $N$ not a perfect square. We highlight that Aut $V_{L_{2 N}}$ for all $N$ has been calculated by Ref. 25, Theorem 2.1 .

Corollary 3.9. For any integer $N$ which is not a perfect square, we have that

$$
\operatorname{Aut}_{(\cdot \cdot)} V_{L_{2 N}}=D_{\infty}
$$

Proof. We have $D_{\infty} \subseteq \operatorname{Aut}_{(\cdot \mid \cdot)} V_{L_{2 N}}$ by Proposition 2.9. Suppose for contradiction there exists $g \in$ Aut $_{(\cdot \mid \cdot)} V_{L_{2 N}} \backslash D_{\infty}$, and let $G$ be the (proper) closed abelian subgroup of $\operatorname{Aut}_{(\cdot \cdot)} V_{L_{2 N}}$ generated by $g$. By the Galois correspondence given in Theorem 3.1 and the fact that $L(1,0)$

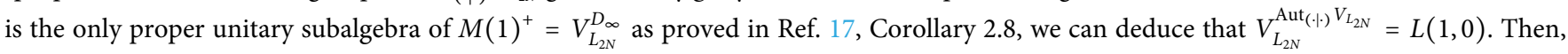


using again the Galois correspondence, $V_{L_{2 N}}^{G}$ is a unitary subalgebra of $V_{L_{2 N}}$ properly containing $L(1,0)$. By Theorem $3.8, V_{L_{2 N}}^{G}$ contains also $M(1)^{+}=V_{L_{2 N}}^{D_{\infty}}$, so $G$ is a subgroup of $D_{\infty}$ by the Galois correspondence, which is a contradiction, so Aut $(\cdot \mid \cdot) V_{L_{2 N}}=D_{\infty}$.

From formula (17), we deduce that for every $N \in \mathbb{Z}_{>0}$, the vector subspace of $V_{L_{2 N}}$ of vectors of conformal weight 2 is spanned by

$$
\begin{aligned}
J_{-2} \Omega \otimes 1, v \otimes 1, J_{-1} \Omega \otimes e^{\sqrt{2} J}, J_{-1} \Omega \otimes e^{-\sqrt{2} J} & (N=1), \\
J_{-2} \Omega \otimes 1, v \otimes 1, \Omega \otimes e^{2 J}, \Omega \otimes e^{-2 J} & (N=2), \\
J_{-2} \Omega \otimes 1, v \otimes 1 & (N>2) .
\end{aligned}
$$

Moreover, it is easy to verify by (18) that

$$
\begin{aligned}
L_{1}\left(J_{-1} \Omega \otimes e^{ \pm \sqrt{2} J}\right) & = \pm \sqrt{2} \Omega \otimes e^{ \pm \sqrt{2} J}, \\
L_{1}\left(J_{-2} \Omega \otimes 1\right) & =2 J_{-1} \Omega \otimes 1, \\
L_{1}(v \otimes 1) & =0, \\
L_{1}\left(\Omega \otimes e^{ \pm 2 J}\right) & =0 .
\end{aligned}
$$

Now, let $W$ be a unitary subalgebra of $V_{L_{2 N}}$. Consider the conformal vector $\omega=e_{W}(v \otimes 1)$ of $W$ as in Proposition 2.2, and let $Y(\omega, z):=\sum_{n \in \mathbb{Z}} L_{n}^{\omega} z^{-n-2}$ be the corresponding energy-momentum field. Using (ii) of Proposition 2.2 together with Ref. 43, Theorem 4.10(iv) and the quasiprimarity of the conformal vector (see Subsection II A), we have that

$$
\begin{aligned}
& L_{0} \omega=L_{0}^{\omega} \omega=2 \omega, \\
& L_{1} \omega=L_{1}^{\omega} \omega=0 .
\end{aligned}
$$

Thus, Eq. (54) imply that $\omega$ must be a quasiprimary vector in $V_{L_{2 N}}$ of conformal weight 2 . Hence, according to (52) and (53), recalling that all nonzero vectors on the right in (53) are linearly independent, $\omega$ must be equal to a multiple of $v \otimes 1$ for $N \neq 2$. Then, we have proved the following result (cf. also Ref. 4, Proposition 5.1):

Proposition 3.10. Let $N \neq 2$. Then, every nontrivial unitary subalgebra of $V_{L_{2 N}}$ contains $L(1,0)$.

Remark 3.11. For $N=k^{2}$ with $k$ as a positive integer, Proposition 3.10 follows also directly from Proposition 2.10 and Theorem 3.2 .

In the following, we investigate the case $N=2$ to be able to complete the classification.

First of all, we know from Ref. 18, Lemma 3.1 (put $2 J=\alpha$ there), cf. also the Proof of Ref. 21 , Theorem 6.3 putting $2 J=\beta$ there, that $V_{L_{4}}$ contains at least two distinct copies of $L\left(\frac{1}{2}, 0\right)$ generated by Virasoro vectors,

$$
\begin{aligned}
& \omega_{0}:=\frac{v \otimes 1}{2}+\frac{\Omega \otimes\left(e^{2 J}+e^{-2 J}\right)}{4}, \\
& \omega_{\pi}:=\frac{v \otimes 1}{2}-\frac{\Omega \otimes\left(e^{2 J}+e^{-2 J}\right)}{4} .
\end{aligned}
$$

Let $W_{0}$ and $W_{\pi}$ be the vertex subalgebras of $V_{L_{4}}$ generated by $\omega_{0}$ and $\omega_{\pi}$, respectively. Then, thanks to Example 2.6 , they are unitary subalgebras of $V_{L_{4}}$, unitarily isomorphic to the unitary Virasoro VOA $L\left(\frac{1}{2}, 0\right)$. Therefore, our goal is to prove the following result.

Theorem 3.12. For every $t \in \mathbb{T}$, the vector

$$
\omega_{t}:=\frac{\nu \otimes 1}{2}+\frac{\Omega \otimes\left(e^{i t} e^{2 J}+e^{-i t} e^{-2 J}\right)}{4} \in V_{L_{4}}
$$

is a Virasoro vector with central charge $\frac{1}{2}$, generating a unitary subalgebra $W_{t} \subset V_{L_{4}}$ unitarily isomorphic to $L\left(\frac{1}{2}, 0\right)$. Moreover, if $W$ is a nontrivial unitary subalgebra of $V_{L_{4}}$ that does not contain $L(1,0)$, then $W=W_{t}=g_{4, t}\left(W_{0}\right)$ for some $t \in \mathbb{T}$. 
To prove Theorem 3.12, we need the following result.

Lemma 3.13. Let $W \subset V_{L_{4}}$ be a nontrivial unitary subalgebra which does not contain $L(1,0)$, then

$$
\omega=e_{W}(v \otimes 1) \in\left\{\omega_{t} \mid t \in \mathbb{T}\right\}
$$

Proof. First of all, $L(1,0) \not \subset W$ implies that the conformal vector $\omega$ of $W$ must be different from $v \otimes 1$. Moreover, it must be different from a multiple of $v \otimes 1$ because $W$ is nontrivial and because it has to satisfy $L_{0}^{\omega} \omega=2 \omega$ as in (54). Consider now the energy-momentum field $Y(\omega, z):=\sum_{n \in \mathbb{Z}} L_{n}^{\omega} z^{-n-2}$ corresponding to $\omega$. Then, (54) implies that $\omega$ must be a linear combination of the three quasiprimary vectors of $V_{L_{4}}$ in (52), that is,

$$
\omega=a v \otimes 1+b \Omega \otimes e^{2 J}+d \Omega \otimes e^{-2 J}
$$

for some $a, b, d \in \mathbb{C}$. Note that either $b \neq 0$ or $d \neq 0$ because $\omega$ is not a multiple of $v \otimes 1$. Using (i) of Proposition 2.2 , namely, $\theta(\omega)=\omega$, we obtain

$$
\omega=a v \otimes 1+\Omega \otimes\left(b e^{2 J}+\bar{b} e^{-2 J}\right)=a v \otimes 1+b e^{2}
$$

for some $a \in \mathbb{R}, b \in \mathbb{C} \backslash\{0\}$, and $e^{2}:=e_{\frac{b}{b}}^{2}=\Omega \otimes\left(e^{2 J}+\frac{\bar{b}}{b} e^{-2 J}\right)$.

We want to calculate $L_{0}^{\omega} \omega$. Consider

$$
\sum_{n \in \mathbb{Z}} L_{n}^{\omega} \omega z^{-n-2}=Y(\omega, z) \omega=a Y(v \otimes 1, z) \omega+b Y\left(e^{2}, z\right) \omega .
$$

Then, we have that

$$
L_{0}^{\omega} \omega=a L_{0} \omega+a b C_{-2, v}+b^{2} C_{-2, e^{2}}=2 a \omega+a b C_{-2, v}+b^{2} C_{-2, e^{2}},
$$

where $C_{-2, v}$ and $C_{-2, e^{2}}$ are $z^{-2}$-coefficients of $Y\left(e^{2}, z\right)(v \otimes 1)$ and $Y\left(e^{2}, z\right) e^{2}$, respectively.

Proceeding as in the Proof of Proposition 3.4, we find

$$
C_{-2, e^{2}}=\frac{\bar{b}}{b} 4\left(J_{-1}\right)^{2} \Omega \otimes 1=\frac{\bar{b}}{b} 8 v \otimes 1
$$

To calculate $C_{-2, v}$, consider

$$
\begin{aligned}
Y\left(\Omega \otimes e^{2 J}, z\right)(v \otimes 1) & =E_{+}(2 J, z) E_{-}(2 J, z) v \otimes e^{2 J}, \\
\frac{\bar{b}}{b} Y\left(\Omega \otimes e^{-2 J}, z\right)(v \otimes 1) & =\frac{\bar{b}}{b} E_{+}(-2 J, z) E_{-}(-2 J, z) v \otimes e^{-2 J},
\end{aligned}
$$

where we have used first Remark 2.8 and second formula (15). Using commutation relations (3) and the fact that $J_{j} \Omega=0$ for all $j \geq 0$ by construction, we get

$$
\begin{aligned}
J_{j}\left(v \otimes e^{ \pm 2 J}\right) & =\frac{1}{2} J_{j}\left(J_{-1}\right)^{2} \Omega \otimes e^{ \pm 2 J}=0 \quad \forall j \geq 2, \\
\left(J_{1}\right)^{j}\left(v \otimes e^{ \pm 2 J}\right) & =\frac{1}{2}\left(J_{1}\right)^{j}\left(J_{-1}\right)^{2} \Omega \otimes e^{ \pm 2 J}=0 \quad \forall j \geq 3, \\
\left(J_{1}\right)^{2}\left(v \otimes e^{ \pm 2 J}\right) & =\frac{1}{2}\left(J_{1}\right)^{2}\left(J_{-1}\right)^{2} \Omega \otimes e^{ \pm 2 J}=\Omega \otimes e^{ \pm 2 J}, \\
J_{1}\left(v \otimes e^{ \pm 2 J}\right) & =\frac{1}{2} J_{1}\left(J_{-1}\right)^{2} \Omega \otimes e^{ \pm 2 J}=J_{-1} \Omega \otimes e^{ \pm 2 J} .
\end{aligned}
$$

Together with (23) and (24), this implies

$$
\begin{aligned}
E_{-}( & \pm 2 J, z)\left(v \otimes e^{ \pm 2 J}\right) \\
& =v \otimes e^{ \pm 2 J}+\frac{-1}{1 !} \cdot \frac{ \pm 2 J_{1}\left(v \otimes e^{ \pm 2 J}\right)}{1} z^{-1}+\frac{(-1)^{2}}{2 !} \cdot \frac{\left( \pm 2 J_{1}\right)^{2}\left(v \otimes e^{ \pm 2 J}\right)}{1 \cdot 1} z^{-2} \\
& =v \otimes e^{ \pm 2 J} \mp\left(2 J_{-1} \Omega \otimes e^{ \pm 2 J}\right) z^{-1}+\left(2 \Omega \otimes e^{ \pm 2 J}\right) z^{-2} .
\end{aligned}
$$


Using (21) and (22), we find

$$
E_{+}( \pm 2 J, z)=I \pm 2 J_{-1} z+O\left(z^{2}\right)
$$

thus,

$$
\begin{aligned}
Y\left(\Omega \otimes e^{2 J}, z\right)(v \otimes 1) & =E_{+}(2 J, z) E_{-}(2 J, z) v \otimes e^{2 J} \\
& =\left(2 \Omega \otimes e^{2 J}\right) z^{-2}+O\left(z^{-1}\right)
\end{aligned}
$$

and

$$
\begin{aligned}
\frac{\bar{b}}{b} Y\left(\Omega \otimes e^{-2 J}, z\right)(v \otimes 1) & =\frac{\bar{b}}{b} E_{+}(-2 J, z) E_{-}(-2 J, z) v \otimes e^{-2 J} \\
& =\frac{\bar{b}}{b}\left(2 \Omega \otimes e^{-2 J}\right) z^{-2}+O\left(z^{-1}\right)
\end{aligned}
$$

We deduce that

$$
C_{-2, v}=2 e^{2} .
$$

We can therefore rewrite identity (59),

$$
L_{0}^{\omega} \omega=2 a \omega+2 a b e^{2}+8|b|^{2} v \otimes 1=2\left(a^{2}+4|b|^{2}\right) v \otimes 1+4 a b e^{2} .
$$

Imposing Eq. (54), namely, $L_{0}^{\omega} \omega=2 \omega$, and using (61), we have the following identity:

$$
2 a v \otimes 1+2 b e^{2}=2\left(a^{2}+4|b|^{2}\right) v \otimes 1+4 a b e^{2}
$$

which has precisely the solutions

$$
a=\frac{1}{2}, \quad b=\frac{e^{i t}}{4}, \quad t \in \mathbb{T} .
$$

Thus, $\omega \in\left\{\omega_{t}: t \in \mathbb{T}\right\}$.

Proof of Theorem 3.12. First, note that $\omega_{t}=g_{4, t}\left(\omega_{0}\right)$ for all $t \in \mathbb{T}$, where $g_{4, t}$ as in (29) is a unitary automorphism of $V_{L_{4}}$. Thus, $\omega_{t} \in V_{L_{4}}$ are still Virasoro vectors of central charge $\frac{1}{2}$, and by Example 2.6, they generate unitary subalgebras $W_{t}=g_{4, t}\left(W_{0}\right)$ of $V_{L_{4}}$ unitarily isomorphic to $L\left(\frac{1}{2}, 0\right)$.

Second, we have to show that every unitary subalgebra $W \subset V_{L_{4}}$ is of the above type. Lemma 3.13 proves that $\omega=e_{W}(v \otimes 1)$ must be of the form $\omega_{t}$, for some $t \in \mathbb{T}$. Accordingly, $W$ is a unitary vertex operator algebra with conformal vector $\omega_{t}$, and hence, its central charge is $c=\frac{1}{2}$. On the other hand, $L\left(\frac{1}{2}, 0\right)$ is the unique, up to isomorphism, unitary vertex operator algebra with central charge $c=\frac{1}{2}$; see, e.g., Ref. 23. It follows that $W=W_{t}$.

Remark 3.14. Note that every $W_{t}$ is contained in $V_{L_{4}}^{D_{1}^{t}}$ for all $t \in \mathbb{T}$. By Remark 2.11, $D_{1}^{t}=D_{1}^{t+\pi}$ for all $t \in \mathbb{T}$. Moreover, under the identification $V_{L_{4}}^{+}=V_{L_{4}}^{D_{1}}=W_{0} \otimes W_{\pi}$ given by Ref. 18, Lemma 3.1 (ii), we have that $V_{L_{4}}^{D_{1}^{t}}=W_{t} \otimes W_{t+\pi}$ for all $t \in \mathbb{T}$.

Then, we can prove the classification theorem.

Theorem 3.15. The nontrivial unitary subalgebras $W$ of the rank-one lattice VOAs $V_{L_{2 N}}$ are classified as follows. Apart from the Virasoro subalgebra $L(1,0)$, we have the following:

(i) If $N=k^{2}$ for some positive integer $k$, then, after the identification of $V_{L_{2 N}}$ with $V_{L_{2}}^{\mathbb{Z}_{k}}, W=V_{L_{2}}^{H}$ for some closed subgroup $H \subseteq S O(3)$ containing $\mathbb{Z}_{k}$.

(ii) If $N>2$ is not a perfect square, then $W=V_{L_{2 N}}^{H}$ for some closed subgroup $H \subseteq D_{\infty}$.

(iii) If $N=2$, then either $W=V_{L_{4}}^{H}$ for some closed subgroup $H \subseteq D_{\infty}$ or $W=W_{t}$ for some $t \in \mathbb{T}$. 
Proof. First of all, note that $L(1,0) \subset V_{L_{2 N}}$ for all $N \in \mathbb{Z}_{>0}$.

(i) The case $k=1$ is Theorem 3.2. By Proposition 2.10, $V_{L_{2 k^{2}}}=V_{L_{2}}^{\mathbb{Z}_{k}}$ for all $k \in \mathbb{Z}_{>0}$ is a unitary subalgebra of $V_{L_{2}}$. Then, the result follows from the Galois correspondence in Theorem 3.1 and from Theorem 3.2.

(ii) By Proposition 3.10, every unitary subalgebra of $V_{L_{2 N}}$ contains $L(1,0)$ and thus contains also $M(1)^{+}$by Theorem 3.8. On the other hand, $M(1)^{+}=V_{L_{2 N}}^{D_{\infty}}$ by Proposition 2.9, and therefore, the result follows from the Galois correspondence in Theorem 3.1.

(iii) If $L(1,0) \subset W$, then we can proceed as in (ii) above. The other case is covered by Theorem 3.12.

\section{THE CLASSIFICATION IN THE CONFORMAL NET SETTING}

Our aim in this section is to arrive at the analog of Theorem 3.15 for conformal nets. For the sake of completeness, we start by recalling basic definitions about conformal nets. For more details, we refer to Ref. 10, Secs. 1-3 and the references therein.

Let $\mathcal{J}$ be the family of open, connected, nonempty, and nondense subsets (also called intervals) of the unit circle $S^{1}$. For all $I \in \mathcal{J}$, we set $I^{\prime}:=S^{1} \backslash \bar{I}$. A (irreducible) conformal net is a family $\mathcal{A}=(\mathcal{A}(I))_{I \in \mathcal{J}}$ of von Neumann algebras on a fixed separable Hilbert space $\mathcal{H}$, which has the following properties:

- (Isotony). For every $I_{1}, I_{2} \in \mathcal{J}$, if $I_{1} \subseteq I_{2}$, then $\mathcal{A}\left(I_{1}\right) \subseteq \mathcal{A}\left(I_{2}\right)$.

- (Locality). For every $I_{1}, I_{2} \in \mathcal{J}$, if $I_{1} \cap I_{2}=\emptyset$, then $\left[\mathcal{A}\left(I_{1}\right), \mathcal{A}\left(I_{2}\right)\right]=0$.

- (Möbius covariance). Let Möb $\cong \operatorname{PSL}(2, \mathbb{R})$ be the group of Möbius transformations of the circle $S^{1}$. Then, there exists a strongly continuous unitary representation $U$ of Möb on $\mathcal{H}$ such that

$$
U(\gamma) \mathcal{A}(I) U(\gamma)^{-1}=\mathcal{A}(\gamma I)
$$

for all $\gamma \in$ Möb and $I \in \mathcal{J}$.

- (Positivity of the energy). The infinitesimal generator $H$ of the one-parameter rotation subgroup of the representation $U$ (also called conformal Hamiltonian) is a positive operator on $\mathcal{H}$.

- (Existence and uniqueness of the vacuum). Let $\bigvee_{I \in \mathcal{J}} \mathcal{A}(I)$ be the von Neumann algebra generated by $\mathcal{A}(I)$ for all $I \in \mathcal{J}$. Then, there exists a unique (up to a phase) $U$-invariant unit vector $\Omega \in \mathcal{H}$ such that $\Omega$ is cyclic for $\bigvee_{I \in \mathcal{J}} \mathcal{A}(I)$. $\Omega$ is called the vacuum vector of the theory.

- (Conformal covariance). Denote by $\operatorname{Diff}^{+}\left(S^{1}\right)$ the group of orientation-preserving diffeomorphisms of the unit circle. For all $I \in \mathcal{J}$, let $\operatorname{Diff}(I) \subset \operatorname{Diff}^{+}\left(S^{1}\right)$ be the subgroup of all $\gamma \in \operatorname{Diff}^{+}\left(S^{1}\right)$ such that $\gamma(z)=z$ for all $z \in I^{\prime}$. Then, there exists a strongly continuous projective unitary representation of $\operatorname{Diff}^{+}\left(S^{1}\right)$ which extends $U$ and such that (we use the same symbol $U$ for the extension)

$$
\begin{aligned}
U(\gamma) \mathcal{A}(I) U(\gamma)^{-1} & =\mathcal{A}(\gamma I) \quad \forall \gamma \in \operatorname{Diff}^{+}\left(S^{1}\right), \\
U(\gamma) A U(\gamma)^{-1} & =A \quad \forall A \in \mathcal{A}(I), \forall \gamma \in \operatorname{Diff}\left(I^{\prime}\right) .
\end{aligned}
$$

To be precise, a conformal net should actually be defined as a triple $(\mathcal{A}, U, \Omega)$ satisfying the above properties. It can be shown that $U$ is completely determined by the pair $(\mathcal{A}, \Omega)$; see, e.g., Ref. 10 , Sec. 3.3 so that a conformal net may be identified with the pair $(\mathcal{A}, \Omega)$. However, we follow the usual convention to denote the conformal net simply by $\mathcal{A}$ keeping in mind all the relevant structures in the definition.

A conformal subnet of $\mathcal{A}$ is a family $\mathcal{B}=(\mathcal{B}(I))_{I \in \mathcal{J}}$ of von Neumann algebras on $\mathcal{H}$ such that

- $\mathcal{B}(I) \subseteq \mathcal{A}(I)$ for all $I \in \mathcal{J}$;

- if $I_{1}, I_{2} \in \mathcal{J}$ with $I_{1} \subseteq I_{2}$, then $\mathcal{B}\left(I_{1}\right) \subseteq \mathcal{B}\left(I_{2}\right)$;

- $U(\gamma) \mathcal{B}(I) U(\gamma)^{-1}=\mathcal{B}(\gamma I)$ for all $\gamma \in$ Möb and $I \in \mathcal{J}$.

Strictly speaking, $\mathcal{B}$ is not a conformal net because $\Omega$ is not a cyclic vector for $\mathcal{B}$ with respect to the Hilbert space $\mathcal{H}$. However, $\mathcal{B}$ becomes a conformal net after restricting to the closure $\mathcal{H}_{\mathcal{B}}$ of $\left(\bigvee_{I \in \mathcal{J}} \mathcal{B}(I)\right) \Omega$.

We define the group of automorphisms of a net $\mathcal{A}$ as

$$
\text { Aut } \mathcal{A}:=\left\{g \in B(\mathcal{H}) \mid g(\Omega)=\Omega, \quad g \mathcal{A}(I) g^{-1}=\mathcal{A}(I) \quad \forall I \in \mathcal{J}\right\} .
$$

It follows from Ref. 51 that Aut $\mathcal{A}$ is a compact topological group with respect to the strong topology induced by $B(\mathcal{H})$. Let $G \subseteq$ Aut $\mathcal{A}$ be a compact subgroup. Then, $\mathcal{A}^{G}$ defined by

$$
\mathcal{A}^{G}(I):=\left\{A \in \mathcal{A}(I) \mid g A g^{-1}=A \quad \forall g \in G\right\}
$$

is a conformal subnet of $\mathcal{A}$ called a fixed point subnet. If $G$ is a finite group, one calls $\mathcal{A}^{G}$ an orbifold subnet. 
The core ingredient of the classification proof in this section is the relationship between conformal nets and unitary VOAs established in Ref. 10. The idea there was to start with a simple unitary $\operatorname{VOA}(V, \Omega, Y, T, v,(\cdot \mid \cdot))$ and to try to construct a conformal net $\left(\mathcal{A}_{V}(I)\right)_{I \in \mathcal{J}}$ on the Hilbert space completion $\mathcal{H}$ of $V$ as follows:

$$
\mathcal{A}_{V}(I):=W^{*}\left\{Y(a, f) \mid a \in V, f \in C^{\infty}\left(S^{1}, \mathbb{R}\right), \quad \operatorname{supp} f \subset I\right\}
$$

where $W^{*}\{\cdot\}$ indicates the smallest von Neumann algebra affiliated with a family of closed densely defined operators $Y(a, f)$ called smeared fields (see Ref. 10, Sec. 2.2). Under an additional technical assumption on the VOA called strong locality, all involved elements are well-defined and the construction works and does indeed define a conformal net, cf. Ref. 10, Theorem 6.8. Furthermore, by Ref. 10, Theorem 6.9, we have the following equality:

$$
\operatorname{Aut}_{(\cdot \cdot)} V=\operatorname{Aut} \mathcal{A}_{V}
$$

More precisely, the extension by continuity of unitary automorphisms of $V$ to the Hilbert space completion $\mathcal{H}$ gives rise to a group isomorphism from $\operatorname{Aut}_{(\cdot \cdot \cdot)} V$ onto Aut $\mathcal{A}_{V}$.

Without the assumption of strong locality, it is unclear whether or not one can define a conformal net in this way. Some of the key results from Ref. 10 we are going to use are the following, cf. Ref. 10, Theorems 7.1, 7.5, and Proposition 7.6:

Theorem 4.1. Let $W$ be a unitary subalgebra of a strongly local simple unitary VOA $V$. Then, $W$ is still strongly local and simple. Furthermore, there is a one-to-one correspondence between unitary subalgebra of $V$ and conformal subnets of $\mathcal{A}_{V}$ given by the map $W \mapsto \mathcal{A}_{W}$.

Proposition 4.2. Let $(V,(\cdot \mid \cdot))$ be a strongly local simple unitary VOA and $G$ be a closed subgroup of Aut $(\cdot \cdot) V=$ Aut $\mathcal{A}_{V}$. Then, $\mathcal{A}_{V}^{G}=\mathcal{A}_{V^{G}}$.

Let us now look at the models we are investigating in this paper. We write $\mathcal{A}_{\mathrm{U}(1)}$ for the $U(1)$-current (conformal) net, and for every positive integer $N \geq 1$, let $\mathcal{A}_{\mathrm{U}(1)_{2 N}}$ denote the extension conformal net of $\mathcal{A}_{\mathrm{U}(1)}$ built from the current $J(z)$, as described in Ref. 2, Proposition 4.1. We call it an even rank-one lattice conformal net. We point out that such an extension is maximal if and only if $N$ is not a

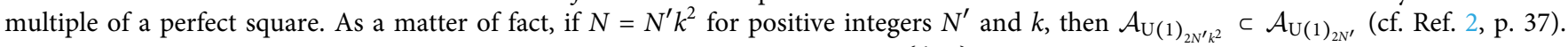
We indicate with $\mathcal{A}_{\mathfrak{V i r}, c}$ the Virasoro (conformal) net with central charge $c \in\left\{\frac{1}{2}, 1\right\}$. Then, Ref. 10, Example 8.8 can be summarized as follows:

Proposition 4.3. For every positive integer $N$, the rank-one lattice type VOA $V_{L_{2 N}}$ is strongly local and $\mathcal{A}_{V_{L_{2 N}}}=\mathcal{A}_{\mathrm{U}(1)_{2 N}}$.

In the following, combining Propositions 4.3 and 4.2, and Theorem 4.1, we can translate the results obtained for rank-one lattice type VOAs in Secs. II B and III, especially Theorem 3.15, to the conformal net setting.

First, by (68) there is a well-defined action of $D_{\infty}$ on $\mathcal{A}_{\mathrm{U}(1)_{2 N}}$, for every $N \in \mathbb{Z}_{>0}$. Thus, we have that $\mathcal{A}_{\mathrm{U}(1)_{2 N k^{2}}}=\mathcal{A}_{\mathrm{U}(1)_{2 N}}^{\mathbb{Z}_{k}}$ for all positive integers $N$ and $k$, thanks to (36). Furthermore, we have a well-defined action of $\mathrm{SO}(3)$ on $\mathcal{A}_{\mathrm{U}(1)_{2}}$ which extends the one of $D_{\infty}$. Set $\mathcal{A}_{t}:=$ $\mathcal{A}_{W_{t}} \cong \mathcal{A}_{\mathfrak{V} \mathfrak{i r}, \frac{1}{2}}$ for all $t \in \mathbb{T}$ with $W_{t}$ as in Theorem 3.12. Then, we can state the following classification result:

Theorem 4.4. The nontrivial conformal subnets $\mathcal{A}$ of the even rank-one lattice conformal nets $\mathcal{A}_{U(1)_{2 N}}$ are classified as follows. Apart from the Virasoro conformal net $\mathcal{A}_{\mathfrak{V i r}, 1}$, we have the following:

(i) If $N=k^{2}$ for some positive integer $k$, then, after the identification $\mathcal{A}_{\mathrm{U}(1)_{2 k^{2}}}=\mathcal{A}_{\mathrm{U}(1)_{2}}^{\mathbb{Z}_{k}}, \mathcal{A}=\mathcal{A}_{\mathrm{U}(1)_{2}}^{H}$ for some closed subgroup $H \subseteq S O(3)$ containing $\mathbb{Z}_{k}$.

(ii) If $N>2$ is not a perfect square, then $\mathcal{A}=\mathcal{A}_{U(1)_{2 N}}^{H}$ for some closed subgroup $H \subseteq D_{\infty}$.

(iii) If $N=2$, then either $\mathcal{A}=\mathcal{A}_{U(1)_{4}}^{H}$ for some closed subgroup $H \subseteq D_{\infty}$ or $\mathcal{A}=\mathcal{A}_{t}$ for some $t \in \mathbb{T}$.

Regarding the case $N=1$, recall that the classification has been known for a while, cf. Ref. 4, Theorem 3.2 and Ref. 53 , Proposition 5 . Recall also that $\mathcal{A}_{\mathrm{U}(1)_{2}}$ is isomorphic to the level 1 loop group net $\mathcal{A}_{\mathrm{SU}(2)_{1}}$ (see Ref. 2, Sec. 5B).

Let $\mathcal{A}_{\mathrm{U}(1)_{2 N}}^{+}:=\mathcal{A}_{\mathrm{U}(1)_{2 N}}^{D_{1}}$ and $\mathcal{A}_{\mathrm{U}(1)}^{+}:=\mathcal{A}_{\mathrm{U}(1)}^{D_{1}}$, and then, we have the conformal net results corresponding to (31), (32), and (37) and Remark 2.11, namely, 


$$
\begin{aligned}
\mathcal{A}_{\mathrm{U}(1)_{2 \mathrm{~N}}}^{\mathbb{T}} & =\mathcal{A}_{\mathrm{U}(1)}, \\
\mathcal{A}_{\mathrm{U}(1)_{2 \mathrm{~N}}}^{D_{\infty}} & =\mathcal{A}_{\mathrm{U}(1)}^{+}, \\
\mathcal{A}_{\mathrm{U}(1)_{2 \mathrm{~N}}}^{D_{k}} & =\mathcal{A}_{\mathrm{U}(1)_{2 N k^{2}},}^{+} \\
\mathcal{A}_{\mathrm{U}(1)_{2 \mathrm{~N}}}^{D_{k}} & =g_{2 N, t} \mathcal{A}_{\mathrm{U}(1)_{2 N \mathrm{k}}}^{+}\left(g_{2 N, t}\right)^{-1} .
\end{aligned}
$$

We can summarize the correspondence between some of the subtheories of rank-one lattice type models in the VOA and in the conformal net setting with the following diagram:

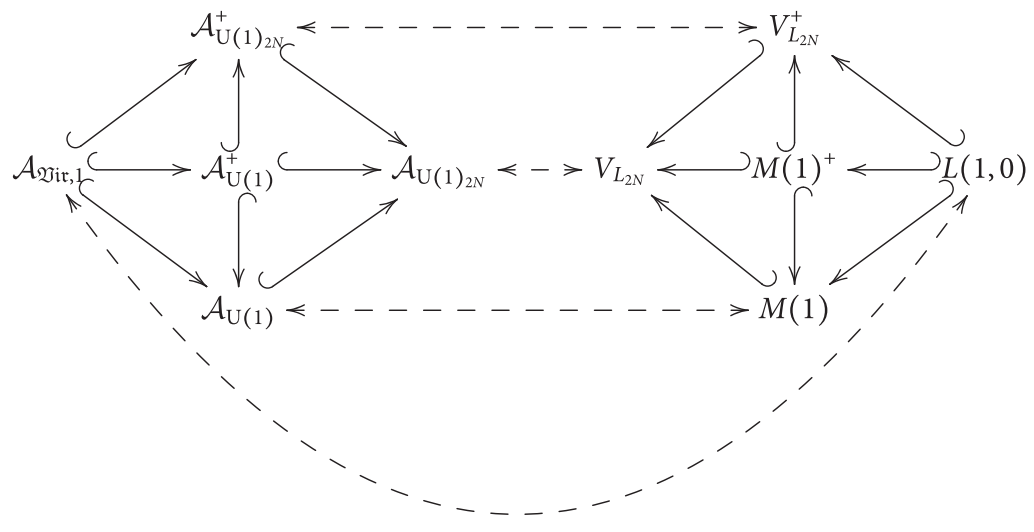

\section{ACKNOWLEDGMENTS}

S.C. would like to thank Yasuyuki Kawahigashi for some useful explanations. INDAM.

S.C. is supported in part by the ERC advanced Grant No. 669240 QUEST "Quantum Algebraic Structures and Models" and GNAMPA-

\section{REFERENCES}

${ }^{1}$ M. Bischoff, "Generalized orbifold construction for conformal nets," Rev. Math. Phys. 29(1), 1750002 (2017).

${ }^{2}$ D. Buchholz, G. Mack, and I. T. Todorov, “The current algebra on the circle as a germ of local field theories," Nucl. Phys. B, Proc. Suppl. 5, 20-56 (1988).

${ }^{3}$ S. Carpi, "Absence of subsystems for the Haag-Kastler nets generated by the energy-momentum tensor in two-dimensional conformal field theory," Lett. Math. Phys. 45, 259-267 (1998).

${ }^{4}$ S. Carpi, "Classification of subsystems for the Haag-Kastler nets generated by c $=1$ chiral current algebras," Lett. Math. Phys. 47(4), 353-364 (1999).

${ }^{5}$ S. Carpi, "On the representation theory of Virasoro nets," Commun. Math. Phys. 244(2), 261-284 (2004).

${ }^{6}$ S. Carpi and R. Conti, "Classification of subsystems for local nets with trivial superselection structure," Commun. Math. Phys. 217(1), 89-106 (2001).

${ }^{7}$ S. Carpi and R. Conti, "Classification of subsystems, local symmetry generators and intrinsic definition of local observables," in Proceedings of the Conference on Mathematical Physics in Mathematics and Physics (Siena 2000), Fields Institute Communications, edited by R. Longo (AMS, 2001), Vol. 30, pp. 83-103.

${ }^{8}$ S. Carpi and R. Conti, “Classification of subsystems for graded-local nets with trivial superselection structure," Commun. Math. Phys. 253, 423-449 (2005).

${ }^{9}$ S. Carpi, Y. Kawahigashi, and R. Longo, "On the Jones index values for conformal subnets," Lett. Math. Phys. 92(2), 98-108 (2010).

${ }^{10}$ S. Carpi, Y. Kawahigashi, R. Longo, and M. Weiner, "From vertex operator algebras to conformal nets and back," Mem. Am. Math. Soc. 254, 1213 (2018).

${ }^{11}$ S. Carpi, M. Weiner, and F. Xu, "From vertex operator algebra modules to representations of conformal nets" (unpublished).

${ }^{12}$ R. Conti, S. Doplicher, and J. E. Roberts, "Superselection theory for subsystems," Commun. Math. Phys. 218(2), 263-281 (2001).

${ }^{13}$ S. Del Vecchio and L. Giorgetti, "Infinite index extensions of local nets and defects," Rev. Math. Phys. 30(2), 1850002 (2017).

${ }^{14}$ P. Di Francesco, P. Mathieu, and D. Sénéchal, Conformal Field Theory (Springer, New York, 1997).

${ }^{15}$ R. Dijkgraaf, C. Vafa, E. Verlinde, and H. Verlinde, “The operator algebra of orbifold models,” Commun. Math. Phys. 123(3), 485-526 (1989).

${ }^{16}$ C. Dong, "Vertex algebras associated with even lattices," J. Algebra 161(1), 242-265 (1993).

${ }^{17}$ C. Dong and R. L. Griess, "Rank one lattice type vertex operator algebras and their automorphism groups," J. Algebra 208(1), 262-275 (1998).

${ }^{18}$ C. Dong, R. L. Griess, and G. Höhn, “Framed vertex operator algebras, codes and the moonshine module," Commun. Math. Phys. 193(2), 407-448 (1998).

${ }^{19} \mathrm{C}$. Dong and C. Jiang, "A characterization of the rational vertex operator algebra $V_{\mathbb{Z} \alpha}^{+}:$II," Adv. Math. 247, 41-70 (2013).

${ }^{20}$ C. Dong and C. Jiang, "A characterization of the rational vertex operator algebra $V_{\mathbb{Z} \alpha}^{+}:$II," J. Reine Angew. Math. 709, 51-79 (2015).

${ }^{21}$ C. Dong, G. Mason, and Y. Zhu, "Discrete series of the Virasoro algebra and the moonshine module," Proc. Symp. Pure Math. 56(2), 295-316 (1994).

${ }^{22}$ C. Dong and X. Lin, "Unitary vertex operator algebras," J. Algebra 397, 252-277 (2014).

${ }^{23}$ C. Dong and X. Lin, "The extensions of $L_{s l_{2}}(k, 0)$ and preunitary vertex operator algebras with central charge c $<1$," Commun. Math. Phys. 340, 613-637 (2015).

${ }^{24}$ C. Dong and G. Mason, "Quantum Galois theory for compact Lie groups," J. Algebra 214, 92-102 (1999). 
${ }^{25}$ C. Dong and K. Nagatomo, "Automorphism groups and twisted modules for lattice vertex operator algebras," in Recent Developments in Quantum Affine Algebras and Related Topics, Contemporary Mathematics (AMS, 1999), Vol. 248, pp. 117-133.

${ }^{26}$ C. Dong and F. Xu, "Conformal nets associated with lattices and their orbifolds," Adv. Math. 206(1), 279-306 (2006).

${ }^{27}$ S. Doplicher and J. E. Roberts, "Endomorphisms of $C^{*}$-algebras, cross products and duality for compact groups," Ann. Math. 130, 75-119 (1989).

${ }^{28}$ S. Doplicher and J. E. Roberts, “A new duality theory for compact groups," Invent. Math. 98, 157-218 (1989).

${ }^{29}$ S. Doplicher and J. E. Roberts, "Why there is a field algebra with a compact gauge group describing the superselection structure in particle physics," Commun. Math. Phys. 131, 51-107 (1990).

${ }^{30}$ D. E. Evans and T. Gannon, “The exoticness and realisability of twisted Haagerup-Izumi modular data," Commun. Math. Phys. 307(2), 463-512 (2011).

${ }^{31}$ D. E. Evans and Y. Kawahigashi, Quantum Symmetries on Operator Algebras (Oxford University Press, New York, 1998).

${ }^{32}$ I. B. Frenkel, Y.-Z. Huang, and J. Lepowsky, “On axiomatic approaches to vertex operator algebras and modules,” Mem. Am. Math. Soc. 104, 494 (1993).

${ }^{33}$ I. B. Frenkel, J. Lepowsky, and A. Meurman, Vertex Operator Algebras and the Monster (Academic Press, Inc., London, 1988).

${ }^{34} \mathrm{~T}$. Gannon, Moonshine Beyond the Monster: The Bridge Connecting Algebra, Modular Forms and Physics (Cambridge University Press, Cambridge, 2006).

${ }^{35}$ P. Ginsparg, "Curiosities at $c=1$," Nucl. Phys. B 295(2), 153-170 (1988).

${ }^{36}$ R. Goodman and N. R. Wallach, "Structure and unitary cocycle representations of loop groups and the group of diffeomorphisms of the circle," J. Reine Angew. Math. 347, 69-133 (1984).

${ }^{37}$ B. Gui, "Unitarity of the modular tensor category associated to unitary vertex operator algebras. I," Commun. Math. Phys. 366, 333-396 (2019).

${ }^{38} \mathrm{~B}$. Gui, "Unitarity of the modular tensor category associated to unitary vertex operator algebras. II," Commun. Math. Phys. (published online); e-print arXiv:1712.04931.

${ }^{39}$ R. Haag, Local Quantum Physics, 2nd ed. (Springer-Verlag, Berlin, Heidelberg, New York, 1996).

${ }^{40}$ N. Jacobson, Lie Algebras (Dover Publications, Inc., New York, 1979).

${ }^{41}$ V. F. R. Jones and F. Xu, "Intersections of finite families of finite index subfactors," Int. J. Math. 15, 717-733 (2004).

${ }^{42}$ V. G. Kac, Infinite Dimensional Lie Algebras (Press Syndicate of the University of Cambridge, Cambridge, 1995).

${ }^{43}$ V. G. Kac, Vertex Algebras for Beginners, University Lecture Notes Vol. 10 (American Mathematical Society, Providence, RI, 2001).

${ }^{44}$ V. G. Kac and A. K. Raina, "Bombay lectures on highest weight representations of infinite dimensional Lie algebras," in Advanced Series in Mathematical Physics (World Scientific Publishing Co. Pte., Ltd., Singapore, 1987), Vol. 2.

${ }^{45}$ Y. Kawahigashi, "Conformal field theory, tensor categories and operator algebras," J. Phys. A: Math. Theor. 48(30), 303001 (2015).

${ }^{46}$ Y. Kawahigashi and R. Longo, "Classification of local conformal nets. Case c < 1," Ann. Math. 160(2), 493-522 (2004).

${ }^{47}$ E. B. Kiritsis, "Proof of the completeness of the classification of rational conformal theories with c = 1," Phys. Lett. B 217, 427-430 (1989).

${ }^{48}$ J. Lepowsky and H. Li, "Introduction to vertex operator algebras and their representations," in Progress in Mathematics (Springer Science+Business Media New York, Birkhäuser Boston, 2004), Vol. 227.

${ }^{49}$ R. Longo, "Conformal subnets and intermediate subfactors," Commun. Math. Phys. 237, 7-30 (2003).

${ }^{50}$ R. Longo and K.-H. Rehren, "Nets of subfactors," Rev. Math. Phys. 7, 567-597 (1995).

${ }^{51}$ V. Morinelli, Y. Tanimoto, and M. Weiner, “Conformal covariance and the split property," Commun. Math. Phys. 357, 379-406 (2018).

${ }^{52}$ A. Pressley and G. Segal, Loop Groups (Oxford University Press, New York, 1986).

${ }^{53}$ K.-H. Rehren, “A new view of the Virasoro algebra," Lett. Math. Phys. 30(2), 125-130 (1994).

${ }^{54}$ J. Tener, "Geometric realization of algebraic conformal field theories," e-print arXiv:1611.01176.

${ }^{55}$ J. Tener, "Representation theory in chiral conformal field theory: From fields to observables," Adv. Math. 349, 488-563 (2019); e-print arXiv:1810.08168.

${ }^{56}$ F. Xu, "Strong additivity and conformal nets," Pac. J. Math. 221(1), 167-199 (2005).

${ }^{57} \mathrm{~F}$. Xu, “On intermediate conformal nets," J. Reine Angew. Math. 692, 125-151 (2014). 